\title{
Ulam-Hyers Stability of Caputo-Type Fractional Stochastic Differential Equations with Time Delays
}

\author{
Xue Wang $\mathbb{D}^{1},{ }^{1}$ Danfeng Luo $\mathbb{D}^{1},{ }^{1}$ Zhiguo Luo $\mathbb{D}^{2},{ }^{2}$ and Akbar Zada $\mathbb{D}^{3}$ \\ ${ }^{1}$ Department of Mathematics, Guizhou University, Guiyang 550025, China \\ ${ }^{2}$ Key Laboratory of Computing and Stochastic Mathematics (Ministry of Education), School of Mathematics and Statistics, \\ Hunan Normal University, Changsha 410081, China \\ ${ }^{3}$ Department of Mathematics, University of Peshawar, Peshawar 25000, Pakistan
}

Correspondence should be addressed to Danfeng Luo; luodf0916@sohu.com

Received 2 February 2021; Revised 11 March 2021; Accepted 17 March 2021; Published 30 March 2021

Academic Editor: FRANCISCO UREÑA

Copyright (C) 2021 Xue Wang et al. This is an open access article distributed under the Creative Commons Attribution License, which permits unrestricted use, distribution, and reproduction in any medium, provided the original work is properly cited.

\begin{abstract}
In this paper, we study a class of Caputo-type fractional stochastic differential equations (FSDEs) with time delays. Under some new criteria, we get the existence and uniqueness of solutions to FSDEs by Caratheodory approximation. Furthermore, with the help of Hölder's inequality, Jensen's inequality, Itô isometry, and Gronwall's inequality, the Ulam-Hyers stability of the considered system is investigated by using Lipschitz condition and non-Lipschitz condition, respectively. As an application, we give two representative examples to show the validity of our theories.
\end{abstract}

\section{Introduction}

The fractional-order differential equations can better simulate many natural physical processes than integerorder differential equations, so it gradually becomes a powerful tool to analyze and solve problems in modern science and technology with the continuous development of natural science and production technology. It is mainly used in the fields of economy and insurance, the analysis of the quantitative structure of biological population, the control of diseases, and the research of genetic law, and we can see these monographs in [1-5]. For more notable achievements of this concept, the readers can also refer to [6-15].

As it is well known, stochastic disturbance is inevitable in practical systems, and it has an important influence on the stability of systems. In [16], $\mathrm{d} u(t)=k u(t) \mathrm{d} t$ was unstable when $k>0$, but it increased the stochastic feedback control $r u(t) \mathrm{d} W(t)$ to become $\mathrm{d} u(t)=k u(t) \mathrm{d} t+r u(t) \mathrm{d} W(t)$. Apparently, $\mathrm{d} u(t)=k u(t) \mathrm{d} t+r u(t) \mathrm{d} W(t)$ was stable if and only if $r^{2}>2 k$. This fact indicated that the stochastic control $r u(t) \mathrm{d} W(t)$ can stabilize the unstable system $\mathrm{d} u(t)=k u(t)$ $\mathrm{d} t$. Therefore, it is significant and challenging to study stochastic stabilization of deterministic systems. More relevant results can be found in [17-19].

The research on the existence and uniqueness of solutions to fractional differential equations is an important content of differential equations. At the same time, the existence and uniqueness have made rapid development in the field of applied mathematics. In [20], the authors studied the existence and uniqueness of positive solutions of some nonlinear fractional differential equations by using mixed monotone operators on cones. Under a number of new conditions and combined with the generalized Gronwall inequality, the uniqueness of solution for fractional $\psi$-Hilfer differential equation with time delays was investigated in [21]. In addition, for many other relevant conclusions, readers can refer to [22-25].

In 1940, S. M. Ulam proposed the stability to functional equations in a speech at the Wisconsin University [26]. 
Hyers [27] was the first to answer the question in 1941. From then, the Ulam-Hyers stability was produced. At the same time, more and more people were interested in exploring the Ulam-Hyers stability. In [28], by using fractional calculus, the properties of classical and generalized Mittag-Leffler functions and the Ulam-Hyers stability of linear fractional differential equations were proved by utilizing the Laplace transform method. The authors investigated the Ulam-Hyers stability, generalized Ulam-Hyers stability, Ulam-Hyers-Rassias stability, and generalized Ulam-Hyers-Rassias stability of impulsive integrodifferential equations with Riemann-Liouville boundary conditions in [29]. For more researched results, we can pay attention to [30-34].

Inspired by the abovementioned, in this article, we are concerned with the existence and Ulam-Hyers stability of Caputo-type FSDEs with time delays:

$$
\begin{cases}{ }^{C} D_{0^{+}}^{\alpha} X(t)=f(t, X(t), X(t-\tau))+g(t, X(t), X(t-\tau)) \frac{\mathrm{d} W(t)}{\mathrm{d} t}, & t \in J=[0, T], \\ X(t)=\Phi(t), & t \in[-\tau, 0],\end{cases}
$$

where $X(0)=\Phi_{0},(1 / 2)<\alpha<1, f:[0, T] \times \mathbb{R}^{d} \times \mathbb{R}^{d} \longrightarrow \mathbb{R}^{d}$, and $g:[0, T] \times \mathbb{R}^{d} \times \mathbb{R}^{d} \longrightarrow \mathbb{R}^{d \times m}$ are measurable continuous functions, $W(t)$ is an $m$-dimensional Brownian motion on a complete probability space $\{\Omega, \mathscr{F}, \mathbb{P}\}, \Phi(t)$ : $[-\tau, 0] \longrightarrow \mathbb{R}^{d}$ is a continuous function, $\mathbb{E}\|\Phi(t)\|^{2}<\infty$, and $\mathbb{E}$ is the mathematical expectation.

Compared with the research results of $[12,20,21,24$, $25,28,34]$, the major contributions of this paper include at least the following three aspects:

(1) In contrast to $[20,21,28]$, the system we study is more generalized because it has not only the stochastic term but also the delay term.

(2) In the methods we investigate the existence and uniqueness of solutions to FSDEs are more novel than $[24,25]$. In $[24,25]$, to explore the existence and uniqueness, Krasnoselskii's fixed point theorem and Mönch's fixed point theorem, respectively, were used. However, in this paper, we adopt the Caratheodory approximation to investigate the existence and uniqueness.

(3) In the study of various stability or existence and uniqueness of FSDEs, many literatures (see [12, 21, 34]) have used a stronger Lipschitz condition. However, in this paper, we used the weak non-Lipschitz condition to discuss the Ulam-Hyers stability of stochastic differential equations. This is a breakthrough in the exploration of the stability to FSDEs.

The structure of this article is arranged as follows. We present some basic definitions and necessary assumptions in Section 2. In Section 3, by Caratheodory approximation, a number of assumed conditions are established for existence and uniqueness of solutions. Section 4 is devoted to testify stability results for the FSDEs with time delays. Examples are given to certify the application of our findings in Section 5.

\section{Preliminaries}

In this section, we intend to recommend a few basic definitions, lemmas, and some necessary assumptions that will play a key role in the paper.
Let $[c, d](-\infty<c<d<+\infty)$ be a finite interval, and we define the norm of $X(t)=\left(X_{1}(t), X_{2}(t), \ldots, X_{d}(t)\right)^{T} \in \mathbb{R}^{d}$ on $[c, d]$ as follows:

$$
\|X(t)\|=\left(\sum_{i=1}^{d} X_{i}^{2}(t)\right)^{(1 / 2)}
$$

Definition 1 (see [35]). The Riemann-Liouville integral operator of fractional-order $\alpha>0$ is defined by

$$
I_{0^{+}}^{\alpha} f(t)=\frac{1}{\Gamma(\alpha)} \int_{0}^{t}(t-v)^{\alpha-1} f(v) \mathrm{d} v,
$$

where $t>0$ and $\Gamma:(0, \infty) \longrightarrow \mathbb{R}$ is the well-known Eulers Gamma function.

Definition 2 (see [1]). For any continuous function $f:[0, T] \times$ $\mathbb{R}^{d} \times \mathbb{R}^{d} \longrightarrow \mathbb{R}^{d}$, the Caputo derivative of fractional-order $\alpha>0$ is defined by

$$
{ }^{C} D_{0^{+}}^{\alpha} f(t)=\frac{1}{\Gamma(n-\alpha)} \int_{0}^{t}(t-v)^{n-\alpha-1} f^{(n)}(v) \mathrm{d} v,
$$

where $n-1<\alpha<n, n \in \mathbb{N}$.

In particular, for $\alpha \in(0,1)$,

$$
I_{0^{+}}^{\alpha C} D_{0^{+}}^{\alpha} f(t)=f(t)-f(0)
$$

Definition 3. An $\mathbb{R}^{d}$-value stochastic process $\{X(t)\}_{-\tau \leq t \leq T}$ is called a solution to equation (1) if it satisfies the following conditions:

(1) $\{X(t)\}$ is $t$-continuous and $\mathscr{F}_{t}$ adapted.

(2) $\{f(t, X(t), X(t-\tau))\} \in L \quad\left([0, T] \times \mathbb{R}^{d} \times \mathbb{R}^{d} ; \mathbb{R}^{d}\right) \mathbb{F}$ and $\{g(t, X(t), X(t-\tau))\} \in L^{2}\left([0, T] \times \mathbb{R}^{d} \times \mathbb{R}^{d}\right.$; $\left.\mathbb{R}^{d \times m}\right)$.

(3) For $\forall t \in[-\tau, T]$, 


$$
X(t)= \begin{cases}\Phi_{0}+\frac{1}{\Gamma(\alpha)} \int_{0}^{t}(t-v)^{\alpha-1} f(v, X(v), X(v-\tau)) \mathrm{d} v & \\ +\frac{1}{\Gamma(\alpha)} \int_{0}^{t} \int_{0}^{t}(t-v)^{\alpha-1} g(v, X(v), X(v-\tau)) \mathrm{d} W(v), & t \in J, \\ \Phi(t), & t \in[-\tau, 0],\end{cases}
$$

where $\mathbb{E}\left(\int_{-\tau}^{T}\|X(t)\|^{2} \mathrm{~d} t\right)<\infty$.

(4) For any other solution $\tilde{X}(t)$, we obtain $P\{X(t)=\widetilde{X}(t),-\tau \leq t \leq T\}=1$.
Definition 4 (see [36]). System (1) is Ulam-Hyers stable if there exists a real number $\delta>0$ such that $\forall \varepsilon>0$ and for each continuously differentiable function $Z(t) \in\left([0, T], \mathbb{R}^{d}\right)$ satisfying

$$
\mathbb{E}\left(\sup _{0 \leq t \leq T}\left\|{ }^{C} D_{0^{+}}^{\alpha} Z(t)-f(t, Z(t), Z(t-\tau))-g(t, Z(t), Z(t-\tau)) \frac{\mathrm{d} W(t)}{\mathrm{d} t}\right\|^{2}\right) \leq \varepsilon,
$$

and there exists a solution $X(t) \in\left([0, T], \mathbb{R}^{d}\right)$ of (1) satisfying

$$
\mathbb{E}\left(\sup _{0 \leq t \leq T}\|Z(t)-X(t)\|^{2}\right)<\varepsilon \delta
$$

Remark 1 (see [21]). A function $Z(t) \in\left([0, T], \mathbb{R}^{d}\right)$ is a solution of equation (7) if and only if there exists a function $h(t) \in\left([0, T], \mathbb{R}^{d}\right)$, such that
(i) $\mathbb{E}\left(\sup \|h(t)\|_{0 \leq t \leq T}^{2}\right) \leq \varepsilon$
(ii) ${ }^{C} D_{0^{+}}^{\alpha} Z(t)=f(t, Z(t), Z(t-\tau))+g(t, Z(t), Z(t-\tau))$ $(\mathrm{d} W(t) / \mathrm{d} t)+h(t)$

Hypothesis 1 (Lipschitz condition). As for any $f, g \in \mathbb{R}^{d}$, there is a constant $l>0$ such that, for all $X_{1}, X_{2}, Y_{1}$, $Y_{2} \in \mathbb{R}^{d}, t \in[0, T]$,

$$
\begin{aligned}
& \left\|f\left(t, X_{1}, Y_{1}\right)-f\left(t, X_{2}, Y_{2}\right)\right\| \vee\left\|g\left(t, X_{1}, Y_{1}\right)-g\left(t, X_{2}, Y_{2}\right)\right\| \\
& \quad \leq l\left(\left\|X_{1}-X_{2}\right\|+\left\|Y_{1}-Y_{2}\right\|\right),
\end{aligned}
$$

where $f$ and $g$ are uniformly continuous functions and $\vee$ is defined as $Y_{1} \vee Y_{2}=\max \left\{Y_{1}, Y_{2}\right\}$.

Hypothesis 2 (non-Lipschitz condition). There is a function $G(t, U, V),[0,+\infty) \times \mathbb{R}^{+} \times \mathbb{R}^{+} \longrightarrow \mathbb{R}^{+}$, such that

(1) For all $X_{1}, X_{2}, Y_{1}, Y_{2} \in \mathbb{R}^{d}$ and $0 \leq t \leq T$,

$$
\begin{aligned}
& \left\|f\left(t, X_{1}, Y_{1}\right)-f\left(t, X_{2}, Y_{2}\right)\right\|^{2} \\
& \quad \vee\left\|g\left(t, X_{1}, Y_{1}\right)-g\left(t, X_{2}, Y_{2}\right)\right\|^{2} \\
& \quad \leq G\left(t,\left\|X_{1}-X_{2}\right\|^{2},\left\|Y_{1}-Y_{2}\right\|^{2}\right),
\end{aligned}
$$

where $f$ and $g$ are continuous as well as bounded functions, and for any fixed $t \geq 0, G(t, U, V)$ is monotone, nondecreasing, continuous, and concave function with $G(t, 0,0)=0$.

(2) For every $t \in \mathbb{R}^{+}$and any nonnegative function $Y(t)$ such that

$$
Y(t) \leq m \int_{0}^{t} G(v, Y(v)) \mathrm{d} v,
$$

where $m>0$ is a constant and $G(v, Y(v), Y(v))=G(v, Y(v))$, we get $Y(t) \equiv 0$.

Hypothesis 3. There exist three functions $a(t), b(t)$, and $q(t)$, such that

$$
\begin{aligned}
& G(t, U, V) \leq a(t)+b(t) U+q(t) V, \quad U, V>0, \\
& \int_{0}^{T} a(t) \mathrm{d} t<\infty, \\
& \int_{0}^{T} b(t) \mathrm{d} t<\infty, \\
& \int_{0}^{T} q(t) \mathrm{d} t<\infty .
\end{aligned}
$$

Lemma 1 (see [37]). Suppose Hypothesis 2 and Hypothesis 3 are fulfilled. Then, there exists constant $c>0$ such that, for any $(t, X, Y) \in[0, T] \times \mathbb{R}^{d} \times \mathbb{R}^{d}$,

$$
\|f(t, X, Y)\|^{2} \vee\|g(t, X, Y)\|^{2} \leq c\left(1+\|X\|^{2}+\|Y\|^{2}\right) .
$$

Proof. Applying Jensen's inequality and Hypothesis 2 and Hypothesis 3, we have 


$$
\begin{aligned}
\| f & (t, X, Y) \|^{2} \\
& =\|f(t, X, Y)-f(t, 0,0)+f(t, 0,0)\|^{2} \\
& \leq 2\|f(t, X, Y)-f(t, 0,0)\|^{2}+2\|f(t, 0,0)\|^{2} \\
& \leq 2 G\left(t,\|X\|^{2},\|Y\|^{2}\right)+2 \sup _{0 \leq t \leq T}\|f(t, 0,0)\|^{2} \\
& \leq 2 a(t)+2 b(t)\|X\|^{2}+2 q(t)\|Y\|^{2}+2 \sup _{0 \leq t \leq T}\|f(t, 0,0)\|^{2} \\
& \leq 2 \sup _{0 \leq t \leq T} a(t)+2 \sup _{0 \leq t \leq T}\|f(t, 0,0)\|^{2}+2 \sup _{0 \leq t \leq T} b(t)\|X\|^{2}+2 \sup _{0 \leq t \leq T} q(t)\|Y\|^{2} \\
& \leq k_{1}\left(1+\|X\|^{2}+\|Y\|^{2}\right),
\end{aligned}
$$

where $k_{1}=\max \left\{2 \sup _{0 \leq t \leq T} a(t)+2 \sup _{0 \leq t \leq T}\|f(t, 0,0)\|^{2}, 2\right.$ $\left.\sup _{0 \leq t \leq T} b(t), 2 \sup _{0 \leq t \leq T} q(t)\right\}<\infty$. In a similar way, we obtain

$$
\|g(t, X, Y)\|^{2} \leq k_{2}\left(1+\|X\|^{2}+\|Y\|^{2}\right) .
$$

Let us set $c=\max \left(k_{1}, k_{2}\right)$. The proof is therefore complete.

\section{Existence and Uniqueness}

Utilizing Caratheodory approximation $[35,38]$, the existence and uniqueness of solutions to SFDEs can be obtained. So, let us define the Caratheodory approximation as follows. For any integer $n \geq 1,0 \leq t \leq T$ define

$$
\begin{aligned}
X_{n}(t)= & \Phi_{0}+\frac{1}{\Gamma(\alpha)} \int_{0}^{t}(t-v)^{\alpha-1} f\left(v, X_{n}\left(v-\frac{1}{n}\right), X_{n}\left((v-\tau)-\frac{1}{n}\right)\right) \mathrm{d} v \\
& +\frac{1}{\Gamma(\alpha)} \int_{0}^{t}(t-v)^{\alpha-1} g\left(v, X_{n}\left(v-\frac{1}{n}\right), X_{n}\left((v-\tau)-\frac{1}{n}\right)\right) \mathrm{d} W(v),
\end{aligned}
$$

and $X_{n}(t)=X(0)=\Phi_{0}$ for all $-(1+\tau) \leq t \leq 0$.

Theorem 1. Suppose that Hypothesis 2 and Hypothesis 3 hold and $3^{(3 / 2)} c T^{2 \alpha-1}<(\alpha-(3 / 4))^{(1 / 2)} \Gamma^{2}(\alpha),(3 / 4)<\alpha<1$; then, system (1) has a unique solution $X(t), t \in[-\tau, T]$.
Proof. The proof will be divided into three steps, when $t \in[0, T]$.

Step 1. The boundedness of the sequence $\left\{X_{n}(t), n \geq 1\right\}$. By (16) and Jensen's inequality, we have

$$
\begin{aligned}
& \mathbb{E}\left(\sup _{0 \leq s \leq t}\left\|X_{n}(s)\right\|^{2}\right) \\
& \leq 3 \mathbb{E}\left\|\Phi_{0}\right\|^{2} \\
& \quad+\frac{3}{\Gamma^{2}(\alpha)} \mathbb{E}\left(\sup _{0 \leq s \leq t}\left\|\int_{0}^{s}(s-v)^{\alpha-1} f\left(v, X_{n}\left(v-\frac{1}{n}\right), X_{n}\left((v-\tau)-\frac{1}{n}\right)\right) \mathrm{d} v\right\|^{2}\right) \\
& +\frac{3}{\Gamma^{2}(\alpha)} \mathbb{E}\left(\sup _{0 \leq s \leq t}\left\|\int_{0}^{s}(s-v)^{\alpha-1} g\left(v, X_{n}\left(v-\frac{1}{n}\right), X_{n}\left((v-\tau)-\frac{1}{n}\right)\right) \mathrm{d} W(v)\right\|^{2}\right) .
\end{aligned}
$$


According to Itô isometry, Cauchy-Schwarz inequality, and Lemma 1 , it is easy to obtain

$$
\begin{aligned}
\mathbb{E}\left(\sup _{0 \leq s \leq t}\left\|X_{n}(s)\right\|^{2}\right) & \leq 3 \mathbb{E}\left\|\Phi_{0}\right\|^{2}+\frac{3}{\Gamma^{2}(\alpha)} \mathbb{E}\left\|\int_{0}^{t}(t-v)^{\alpha-1} f\left(v, X_{n}\left(v-\frac{1}{n}\right), X_{n}\left((v-\tau)-\frac{1}{n}\right)\right) \mathrm{d} v\right\|^{2} \\
& +\frac{3}{\Gamma^{2}(\alpha)} \mathbb{E} \int_{0}^{t}(t-v)^{2 \alpha-2}\left\|g\left(v, X_{n}\left(v-\frac{1}{n}\right), X_{n}\left((v-\tau)-\frac{1}{n}\right)\right)\right\|^{2} \mathrm{~d} v \\
\leq & 3 \mathbb{E}\left\|\Phi_{0}\right\|^{2} \\
& +\frac{3 T^{2 \alpha-1}}{(2 \alpha-1) \Gamma^{2}(\alpha)} \mathbb{E} \int_{0}^{t} c\left(1+\left\|X_{n}\left(v-\frac{1}{n}\right)\right\|^{2}+\left\|X_{n}\left((v-\tau)-\frac{1}{n}\right)\right\|^{2}\right) \mathrm{d} v \\
& +\frac{3}{\Gamma^{2}(\alpha)} \mathbb{E} \int_{0}^{t}(t-v)^{2 \alpha-2} c\left(1+\left\|X_{n}\left(v-\frac{1}{n}\right)\right\|^{2}+\left\|X_{n}\left((v-\tau)-\frac{1}{n}\right)\right\|^{2}\right) \mathrm{d} v \\
= & J_{1}+J_{2}+J_{3} .
\end{aligned}
$$

Letting $v_{1}=v-(1 / n)$ and $v_{2}=v-\tau-(1 / n)$. It is obvious that $v_{1} \in[-1, v], v_{2} \in[-(1+\tau), v]$, and

$$
\begin{aligned}
J_{2} \leq & \frac{3 c T^{2 \alpha-1}}{(2 \alpha-1) \Gamma^{2}(\alpha)} \\
& \cdot \int_{0}^{t}\left(1+\mathbb{E}\left(\sup _{-1 \leq v_{1} \leq v}\left\|X_{n}\left(v_{1}\right)\right\|^{2}\right)+\mathbb{E}\left(\sup _{-(1+\tau) \leq v_{2} \leq v}\left\|X_{n}\left(v_{2}\right)\right\|^{2}\right)\right) \mathrm{d} v \\
\leq & \frac{3 c T^{2 \alpha-1}}{(2 \alpha-1) \Gamma^{2}(\alpha)} \int_{0}^{t}\left(1+2 \mathbb{E}\left(\sup _{-(1+\tau) \leq v_{2} \leq v}\left\|X_{n}\left(v_{2}\right)\right\|^{2}\right)\right) \mathrm{d} v \\
\leq & c_{1}\left[T+2 \int_{0}^{t} \max \left(\mathbb{E}\left(\sup _{-(1+\tau) \leq V_{2} \leq 0}\left\|X_{n}\left(v_{2}\right)\right\|^{2}\right), \mathbb{E}\left(\sup _{0 \leq v_{2} \leq v}\left\|x_{n}\left(v_{2}\right)\right\|^{2}\right)\right) \mathrm{d} v\right] \\
= & c_{1} T+2 c_{1} \int_{0}^{t} \max \left(\mathbb{E}\left\|\Phi_{0}\right\|^{2}, \mathbb{E}\left(\sup _{0 \leq v_{2} \leq v}\left\|X_{n}\left(v_{2}\right)\right\|^{2}\right)\right) \mathrm{d} v,
\end{aligned}
$$


where $c_{1}=\left(3 c T^{2 \alpha-1} /(2 \alpha-1) \Gamma^{2}(\alpha)\right)$.
Using Hölders inequality and Jensen's inequality, we obtain

$$
\begin{aligned}
J_{3} & \leq \frac{3 c}{\Gamma^{2}(\alpha)}\left(\int_{0}^{t}(t-v)^{4 \alpha-4} \mathrm{~d} v\right)^{(1 / 2)} \\
& \mathbb{E}\left(\int_{0}^{t}\left(1+\left\|X_{n}\left(v-\frac{1}{n}\right)\right\|^{2}+\left\|X_{n}\left((v-\tau)-\frac{1}{n}\right)\right\|^{2}\right)^{2} \mathrm{~d} v\right)^{(1 / 2)} \\
& \leq \frac{3 c T^{2 \alpha-(3 / 2)}}{(4 \alpha-3)^{(1 / 2)} \Gamma^{2}(\alpha)} \mathbb{E}\left(\int_{0}^{t}\left(3+3\left\|X_{n}\left(v-\frac{1}{n}\right)\right\|^{4}+3\left\|X_{n}\left((v-\tau)-\frac{1}{n}\right)\right\|^{4}\right) \mathrm{d} v\right)^{(1 / 2)},
\end{aligned}
$$

where $3+3\left\|X_{n}(v-(1 / n))\right\|^{4}+3\left\|X_{n}((v-\tau)-(1 / n))\right\|^{4}$ is continuous function on $[0, t]$. By the mean value theorem of integrals, there exists $y \in[0, t]$ such that

$$
\begin{gathered}
\int_{0}^{t}\left(3+3\left\|X_{n}\left(v-\frac{1}{n}\right)\right\|^{4}+3\left\|X_{n}\left((v-\tau)-\frac{1}{n}\right)\right\|^{4}\right) \mathrm{d} v \\
=t\left(3+3\left\|X_{n}\left(y-\frac{1}{n}\right)\right\|^{4}+3\left\|X_{n}\left((y-\tau)-\frac{1}{n}\right)\right\|^{4}\right)
\end{gathered}
$$

$$
\leq T\left(3+3\left\|X_{n}\left(y-\frac{1}{n}\right)\right\|^{4}+3\left\|X_{n}\left((y-\tau)-\frac{1}{n}\right)\right\|^{4}\right) .
$$

Using the inequality

$$
\sqrt{L_{1}+L_{2}+L_{3}} \leq \sqrt{\left\|L_{1}\right\|}+\sqrt{\left\|L_{2}\right\|}+\sqrt{\left\|L_{3}\right\|},
$$

we derive

$$
\begin{aligned}
J_{3} & \leq \frac{3 c T^{2 \alpha-1}}{(4 \alpha-3)^{(1 / 2)} \Gamma^{2}(\alpha)} \mathbb{E}\left(3+3\left\|X_{n}\left(y-\frac{1}{n}\right)\right\|^{4}+3\left\|X_{n}\left((y-\tau)-\frac{1}{n}\right)\right\|^{4}\right)^{(1 / 2)} \\
& \leq \frac{3^{(3 / 2)} c T^{2 \alpha-1}}{(4 \alpha-3)^{(1 / 2)} \Gamma^{2}(\alpha)} \mathbb{E}\left(1+\left\|X_{n}\left(y-\frac{1}{n}\right)\right\|^{2}+\left\|X_{n}\left((y-\tau)-\frac{1}{n}\right)\right\|^{2}\right) .
\end{aligned}
$$

Let us set $v_{3}=y-(1 / n), v_{4}=y-\tau-(1 / n), v_{3} \in[-1, t]$, and $v_{4} \in[-(1+\tau), t]$, and we have

$$
\begin{aligned}
J_{3} & \leq \frac{3^{(3 / 2)} c T^{2 \alpha-1}}{(4 \alpha-3)^{1 / 2} \Gamma^{2}(\alpha)} \mathbb{E}\left(1+\left\|X_{n}\left(v_{3}\right)\right\|^{2}+\left\|X_{n}\left(v_{4}\right)\right\|^{2}\right) \\
& \leq \frac{3^{(3 / 2)} c T^{2 \alpha-1}}{(4 \alpha-3)^{(1 / 2)} \Gamma^{2}(\alpha)}\left(1+\mathbb{E}\left(\sup _{-1 \leq v_{3} \leq t}\left\|X_{n}\left(v_{3}\right)\right\|^{2}+\mathbb{E}\left(\sup _{-(1+\tau) \leq v_{4} \leq t}\left\|X_{n}\left(v_{4}\right)\right\|^{2}\right)\right)\right. \\
& \leq \frac{3^{(3 / 2)} c T^{2 \alpha-1}}{(4 \alpha-3)^{(1 / 2)} \Gamma^{2}(\alpha)}+\frac{3^{(3 / 2)} c T^{2 \alpha-1}}{(4 \alpha-3)^{(1 / 2)} \Gamma^{2}(\alpha)} 2 \mathbb{E}\left(\sup _{-(1+\tau) \leq V_{4} \leq t}\left\|X_{n}\left(v_{4}\right)\right\|^{2}\right) \\
& =c_{2}+2 c_{2} \max \left(\mathbb{E}\left\|\Phi_{0}\right\|^{2}, \mathbb{E}\left(\sup _{0 \leq v_{4} \leq t}\left\|X_{n}\left(v_{4}\right)\right\|^{2}\right)\right),
\end{aligned}
$$


Mathematical Problems in Engineering

7

where $c_{2}=\left(3^{(3 / 2)} c T^{2 \alpha-1} /(4 \alpha-3)^{(1 / 2)} \Gamma^{2}(\alpha)\right)$.

If $\max \left(\mathbb{E}\left\|\Phi_{0}\right\|^{2}, \mathbb{E}\left(\sup _{0 \leq v_{4} \leq t}\left\|X_{n}\left(v_{4}\right)\right\|^{2}\right)\right)=\mathbb{E}\left\|\Phi_{0}\right\|^{2}$, we obtain

$$
\begin{aligned}
& \mathbb{E}\left(\sup _{0 \leq s \leq t}\left\|X_{n}(s)\right\|^{2}\right) \\
& \quad \leq J_{1}+J_{2}+J_{3} \\
& \quad \leq 3 \mathbb{E}\left\|\Phi_{0}\right\|^{2}+c_{1} T+2 c_{1} \int_{0}^{t} \mathbb{E}\left\|\Phi_{0}\right\|^{2} \mathrm{~d} v+c_{2}+2 c_{2} \mathbb{E}\left\|\Phi_{0}\right\|^{2} \\
& \quad \leq\left(3+2 c_{1} T+2 c_{2}\right) \mathbb{E}\left\|\Phi_{0}\right\|^{2}+c_{1} T+c_{2}=\beta_{1} .
\end{aligned}
$$

If $\max \left(\mathbb{E}\left\|\Phi_{0}\right\|^{2}, \mathbb{E}\left(\sup _{0 \leq v_{2} \leq v}\left\|X_{n}\left(v_{2}\right)\right\|^{2}\right)\right)=\mathbb{E}\left(\sup _{0 \leq v_{2} \leq v}\right.$ $\left.\left\|X_{n}\left(v_{2}\right)\right\|^{2}\right)$, we obtain

$$
\begin{aligned}
\mathbb{E}\left(\sup _{0 \leq s \leq t}\left\|X_{n}(s)\right\|^{2}\right) \\
\leq J_{1}+J_{2}+J_{3} \\
\leq 3 \mathbb{E}\left\|\Phi_{0}\right\|^{2}+c_{1} T+2 c_{1} \int_{0}^{t} \mathbb{E}\left(\sup _{0 \leq v_{2} \leq v}\left\|X_{n}\left(v_{2}\right)\right\|^{2}\right) \mathrm{d} v \\
\quad+c_{2}+2 c_{2} \mathbb{E}\left(\sup _{0 \leq v_{4} \leq t}\left\|X_{n}\left(v_{4}\right)\right\|^{2}\right) \\
=3 \mathbb{E}\left\|\Phi_{0}\right\|^{2}+c_{1} T+c_{2}+2 c_{1} \int_{0}^{t} \mathbb{E}\left(\sup _{0 \leq v_{2} \leq v}\left\|X_{n}\left(v_{2}\right)\right\|^{2}\right) \mathrm{d} v \\
\quad+2 c_{2} \mathbb{E}\left(\sup _{0 \leq s \leq t}\left\|X_{n}(s)\right\|^{2}\right),
\end{aligned}
$$

and then,

$$
\begin{aligned}
\mathbb{E}\left(\sup _{0 \leq s \leq t}\left\|X_{n}(s)\right\|^{2}\right) \\
\leq \frac{3}{1-2 c_{2}} \mathbb{E}\left\|\Phi_{0}\right\|^{2}+\frac{c_{1} T+c_{2}}{1-2 c_{2}}+\frac{2 c_{1}}{1-2 c_{2}} \int_{0}^{t} \mathbb{E} \\
\cdot\left(\sup _{0 \leq v_{2} \leq v} \|\left(X_{n} \|^{2}\right) \mathrm{d} v .\right.
\end{aligned}
$$

By Gronwall's inequality, we can conclude that

$$
\begin{aligned}
& \mathbb{E}\left(\sup _{0 \leq s \leq t}\left\|X_{n}(s)\right\|^{2}\right) \\
& \leq\left(\frac{3}{1-2 c_{2}} \mathbb{E}\left\|\Phi_{0}\right\|^{2}+\frac{c_{1} T+c_{2}}{1-2 c_{2}}\right) e^{\left(2 c_{1} T / 1-2 c_{2}\right)} \\
& =\beta_{2} .
\end{aligned}
$$

Letting $\beta=\max \left(\beta_{1}, \beta_{2}\right)$, we obtain

$$
\mathbb{E}\left(\sup _{0 \leq s \leq t}\left\|X_{n}(s)\right\|^{2}\right) \leq \beta,
$$

where $\beta$ is a positive constant. So, we have proved that the sequence $\left\{X_{n}(t), n \geq 1\right\}$ is bounded.

Step 2. For $0 \leq s<t \leq T$ and any integer $n \geq 1$, we obtain by (16)

$$
\begin{aligned}
X_{n}(t) & -X_{n}(s) \\
= & \frac{1}{\Gamma(\alpha)} \int_{0}^{s}\left((t-v)^{\alpha-1}-(s-v)^{\alpha-1}\right) f\left(v, X_{n}\left(v-\frac{1}{n}\right), X_{n}\left((v-\tau)-\frac{1}{n}\right)\right) \mathrm{d} v \\
& +\frac{1}{\Gamma(\alpha)} \int_{s}^{t}(t-v)^{\alpha-1} f\left(v, X_{n}\left(v-\frac{1}{n}\right), X_{n}\left((v-\tau)-\frac{1}{n}\right)\right) \mathrm{d} v \\
& +\frac{1}{\Gamma(\alpha)} \int_{0}^{s}\left((t-v)^{\alpha-1}-(s-v)^{\alpha-1}\right) g\left(v, X_{n}\left(v-\frac{1}{n}\right), X_{n}\left((v-\tau)-\frac{1}{n}\right)\right) \mathrm{d} W(v) \\
& +\frac{1}{\Gamma(\alpha)} \int_{s}^{t}(t-v)^{\alpha-1} g\left(v, X_{n}\left(n-\frac{1}{n}\right), X_{n}\left((v-\tau)-\frac{1}{n}\right)\right) \mathrm{d} W(v) .
\end{aligned}
$$


By using Jensen's inequality, Cauchy-Schwarz inequality, and Itô isometry, we conclude

$$
\begin{aligned}
\mathbb{E}\left\|X_{n}(t)-X_{n}(s)\right\|^{2} & \frac{4}{\Gamma^{2}(\alpha)} \mathbb{E}\left\|\int_{0}^{s}\left((t-v)^{\alpha-1}-(s-v)^{\alpha-1}\right) f\left(v, X_{n}\left(v-\frac{1}{n}\right), X_{n}\left((v-\tau)-\frac{1}{n}\right)\right) \mathrm{d} v\right\|^{2} \\
& +\frac{4}{\Gamma^{2}(\alpha)} \mathbb{E}\left\|\int_{s}^{t}(t-v)^{\alpha-1} f\left(v, X_{n}\left(v-\frac{1}{n}\right), X_{n}\left((v-\tau)-\frac{1}{n}\right)\right) \mathrm{d} v\right\|^{2} \\
& +\frac{4}{\Gamma^{2}(\alpha)} \mathbb{E}\left\|\int_{0}^{s}\left((t-v)^{\alpha-1}-(s-v)^{\alpha-1}\right) g\left(v, X_{n}\left(v-\frac{1}{n}\right), X_{n}\left((v-\tau)-\frac{1}{n}\right)\right) \mathrm{d} W(v)\right\|^{2} \\
& +\frac{4}{\Gamma^{2}(\alpha)} \mathbb{E}\left\|\int_{s}^{t}(t-v)^{\alpha-1} g\left(v, X_{n}\left(v-\frac{1}{n}\right), X_{n}\left((v-\tau)-\frac{1}{n}\right)\right) \mathrm{d} W(v)\right\|^{2} \\
\leq & \frac{4 T}{\Gamma^{2}(\alpha)} \mathbb{E} \int_{0}^{s} \|\left((t-v)^{\alpha-1}-(s-v)^{\alpha-1}\right) f\left(v, X_{n}\left(v-\frac{1}{n}\right), X_{n}\left((v-\tau)-\frac{1}{n}\right) \|^{2} \mathrm{~d} v\right. \\
& +\frac{4 T}{\Gamma^{2}(\alpha)} \mathbb{E} \int_{s}^{t}\left\|(t-v)^{\alpha-1} f\left(v, X_{n}\left(v-\frac{1}{n}\right), X_{n}\left((v-\tau)-\frac{1}{n}\right)\right)\right\|^{2} \mathrm{~d} v \\
& +\frac{4}{\Gamma^{2}(\alpha)} \mathbb{E} \int_{0}^{s}\left\|\left((t-v)^{\alpha-1}-(s-v)^{\alpha-1}\right) g\left(v, X_{n}\left(v-\frac{1}{n}\right), X_{n}\left((v-\tau)-\frac{1}{n}\right)\right)\right\|^{2} \mathrm{~d} v \\
& +\frac{4}{\Gamma^{2}(\alpha)} \int_{s}^{t}\left\|(t-v)^{\alpha-1} g\left(v, X_{n}\left(v-\frac{1}{n}\right), X_{n}\left((v-\tau)-\frac{1}{n}\right)\right)\right\|^{2} \mathrm{~d} v .
\end{aligned}
$$

Recalling Lemma 1, we obtain

$$
\begin{aligned}
& \mathbb{E}\left\|X_{n}(t)-X_{n}(s)\right\|^{2} \\
& \leq \frac{4 c(1+T)}{\Gamma^{2}(\alpha)} \int_{0}^{s}\left[(t-v)^{\alpha-1}-(s-v)^{\alpha-1}\right]^{2} \mathbb{E}\left[1+\left\|X_{n}\left(v-\frac{1}{n}\right)\right\|^{2}+\left\|X_{n}\left((v-\tau)-\frac{1}{n}\right)\right\|^{2}\right] \mathrm{d} v \\
& \quad+\frac{4 c(1+T)}{\Gamma^{2}(\alpha)} \int_{s}^{t}(t-v)^{2 \alpha-2} \mathbb{E}\left[1+\left\|X_{n}\left(v-\frac{1}{n}\right)\right\|^{2}+\left\|X_{n}\left((v-\tau)-\frac{1}{n}\right)\right\|^{2}\right] \mathrm{d} v \\
& =c_{3}\left(J_{4}+J_{5}\right),
\end{aligned}
$$

where $c_{3}=\left(4 c(1+T) / \Gamma^{2}(\alpha)\right)$. 


$$
\begin{aligned}
& J_{4} \leq\left[\int_{0}^{s}\left((t-v)^{\alpha-1}-(s-v)^{\alpha-1}\right)^{4} \mathrm{~d} v\right]^{(1 / 2)} \\
& \cdot\left[\int_{0}^{s}\left(1+\mathbb{E}\left\|X_{n}\left(v-\frac{1}{n}\right)\right\|^{2}+\mathbb{E}\left\|X_{n}\left((v-\tau)-\frac{1}{n}\right)\right\|^{2}\right)^{2} \mathrm{~d} v\right]^{(1 / 2)} \\
& \leq\left[\int_{0}^{s}\left((t-v)^{\alpha-1}-(s-v)^{\alpha-1}\right)^{4} \mathrm{~d} v\right]^{(1 / 2)}\left[\int _ { 0 } ^ { s } \left(1+\mathbb{E}\left(\sup _{0 \leq u_{1} \leq v}\left\|X_{n}\left(u_{1}-\frac{1}{n}\right)\right\|^{2}\right)\right.\right. \\
& \left.\left.+\mathbb{E}\left(\sup _{0 \leq u_{2} \leq v}\left\|X_{n}\left(\left(u_{2}-\tau\right)-\frac{1}{n}\right)\right\|^{2}\right)\right)^{2} \mathrm{~d} v\right]^{(1 / 2)} \\
& \leq\left[\int_{0}^{s}\left((t-v)^{\alpha-1}-(s-v)^{\alpha-1}\right)^{4} \mathrm{~d} v\right]^{\frac{1}{2}}\left[\int_{0}^{s}(1+2 \beta)^{2} \mathrm{~d} v\right]^{(1 / 2)} \\
& \leq(1+2 \beta) T^{(1 / 2)}\left[\int_{0}^{s}\left((t-v)^{\alpha-1}-(s-v)^{\alpha-1}\right)^{4} \mathrm{~d} v\right]^{(1 / 2)} \\
& =(1+2 \beta) T^{(1 / 2)}\left[\int _ { 0 } ^ { s } \left(\left((t-v)^{2 \alpha-2}+(s-v)^{2 \alpha-2}\right)^{2}+4(t-v)^{2 \alpha-2}(s-v)^{2 \alpha-2}\right.\right. \\
& \left.\left.-4(t-v)^{3 \alpha-3}(s-v)^{\alpha-1}-4(s-v)^{3 \alpha-3}(t-v)^{\alpha-1}\right) \mathrm{~d} v\right]^{(1 / 2)} \\
& \leq(1+2 \beta) T^{(1 / 2)}\left[\int_{0}^{s}\left(2(t-v)^{4 \alpha-4}+2(s-v)^{4 \alpha-4}+4(s-v)^{4 \alpha-4}-8(t-v)^{4 \alpha-4}\right) \mathrm{d} v\right]^{(1 / 2)} \\
& =(1+2 \beta) T^{(1 / 2)}\left[\int_{0}^{s}\left(6(s-v)^{4 \alpha-4}-6(t-v)^{4 \alpha-4}\right) \mathrm{d} v\right]^{(1 / 2)} \\
& =(1+2 \beta)(6 T)^{(1 / 2)}\left[\frac{(t-s)^{4 \alpha-3}}{4 \alpha-3}+\frac{s^{4 \alpha-3}}{4 \alpha-3}-\frac{t^{4 \alpha-3}}{4 \alpha-3}\right]^{(1 / 2)} \\
& \leq(1+2 \beta)(6 T)^{(1 / 2)} \frac{(t-s)^{2 \alpha-(1 / 2)}}{(4 \alpha-3)^{(1 / 2)}} \\
& =c_{4}(t-s)^{2 \alpha-(1 / 2)} \text {, }
\end{aligned}
$$

where $c_{4}=\left((1+2 \beta)(6 T)^{(1 / 2)} /(4 \alpha-3)^{(1 / 2)}\right)$.

Using Hölder's inequality and Step 1 again,

$$
\begin{aligned}
J_{5} & \leq\left[\int_{s}^{t}(t-v)^{4 \alpha-4} \mathrm{~d} v\right]^{(1 / 2)}\left[\int_{s}^{t}(1+2 \beta)^{2} \mathrm{~d} v\right]^{(1 / 2)} \\
& =(t-s)^{(1 / 2)}(1+2 \beta)\left[\int_{s}^{t}(t-v)^{4 \alpha-4} \mathrm{~d} v\right]^{(1 / 2)} \\
& =\frac{(t-s)^{2 \alpha-1}(1+2 \beta)}{(4 \alpha-3)^{(1 / 2)}} \\
& =c_{5}(t-s)^{2 \alpha-1}
\end{aligned}
$$

where $c_{5}=\left((1+2 \beta) /(4 \alpha-3)^{(1 / 2)}\right)$.

Then,

$$
\begin{aligned}
\mathbb{E}\left\|X_{n}(t)-X_{n}(s)\right\|^{2} & \leq c_{3}\left(J_{4}+J_{5}\right) \\
& \leq c_{3} c_{4}(t-s)^{2 \alpha-(3 / 2)}+c_{3} c_{5}(t-s)^{2 \alpha-1} \\
& =r_{1}(t-s)^{2 \alpha-(3 / 2)}+r_{2}(t-s)^{2 \alpha-1},
\end{aligned}
$$

where $r_{1}=c_{3} c_{4}$ and $r_{2}=c_{3} c_{5}$.

Step 3. We claim that $\left\{X_{n}(t), n \geq 1\right\}$ is a Cauchy sequence. For integer $m>n \geq 1$, one can obtain 
10

Mathematical Problems in Engineering

$$
\begin{aligned}
X_{m}(s)-X_{n}(s)= & \frac{1}{\Gamma(\alpha)} \int_{0}^{s}(s-v)^{\alpha-1}\left[f\left(v, X_{m}\left(v-\frac{1}{m}\right), X_{m}\left((v-\tau)-\frac{1}{m}\right)\right)\right. \\
& \left.-f\left(v, X_{n}\left(v-\frac{1}{n}\right), X_{n}\left((v-\tau)-\frac{1}{n}\right)\right)\right] \mathrm{d} v \\
& +\frac{1}{\Gamma(\alpha)} \int_{0}^{s}(s-v)^{\alpha-1}\left[g\left(v, X_{m}\left(v-\frac{1}{m}\right), X_{m}\left((v-\tau)-\frac{1}{m}\right)\right)\right. \\
& \left.-g\left(v, X_{n}\left(v-\frac{1}{n}\right), X_{n}\left((v-\tau)-\frac{1}{n}\right)\right)\right] \mathrm{d} W(v) .
\end{aligned}
$$

By Jensen's inequality, Hölder's inequality, and Ito isometry, we can obtain

$$
\begin{aligned}
& \mathbb{E}\left(\sup _{0 \leq s \leq t}\left\|X_{m}(s)-X_{n}(s)\right\|^{2}\right) \\
& \leq \frac{2}{\Gamma^{2}(\alpha)} \mathbb{E} \| \int_{0}^{t}(t-v)^{\alpha-1}\left[f\left(v, X_{m}\left(v-\frac{1}{m}\right), X_{m}\left((v-\tau)-\frac{1}{m}\right)\right)\right. \\
&\left.\quad-f\left(v, X_{n}\left(v-\frac{1}{n}\right), X_{n}\left((v-\tau)-\frac{1}{n}\right)\right)\right] \mathrm{d} v \|^{2} \\
&+\frac{2}{\Gamma^{2}(\alpha)} \mathbb{E} \| \int_{0}^{t}(t-v)^{\alpha-1}\left[g\left(v, X_{m}\left(v-\frac{1}{m}\right), X_{m}\left((v-\tau)-\frac{1}{m}\right)\right)\right. \\
&\left.\quad-g\left(v, X_{n}\left(v-\frac{1}{n}\right), X_{n}\left((v-\tau)-\frac{1}{n}\right)\right)\right] \mathrm{d} W(v) \|^{2} \\
& \leq \frac{2}{\Gamma^{2}(\alpha)} \int_{0}^{t}(t-v)^{2 \alpha-2} \mathrm{~d} v \int_{0}^{t} \mathbb{E} \| f\left(v, X_{m}\left(v-\frac{1}{m}\right), X_{m}\left((v-\tau)-\frac{1}{m}\right)\right) \\
&-f\left(v, X_{n}\left(v-\frac{1}{n}\right), X_{n}\left((v-\tau)-\frac{1}{n}\right)\right) \|^{2} \mathrm{~d} v \\
&= J_{7}+J_{8} . \\
&+\frac{2}{\Gamma^{2}(\alpha)} \int_{0}^{t}(t-v)^{2 \alpha-2} \mathbb{E} \| g\left(v, X_{m}\left(v-\frac{1}{m}\right), X_{m}\left((v-\tau)-\frac{1}{m}\right)\right) \\
&-g\left(X_{n}\left(v-\frac{1}{n}\right), X_{n}\left((v-\tau)-\frac{1}{n}\right)\right) \|^{2} \mathrm{~d} v
\end{aligned}
$$


By applying Jensen's inequality and Hypothesis 2, we obtain

$$
\begin{aligned}
J_{7} \leq & \frac{2 T^{2 \alpha-1}}{(2 \alpha-1) \Gamma^{2}(\alpha)} \int_{0}^{t} \mathbb{E} \| f\left(v, X_{m}\left(v-\frac{1}{m}\right), X_{m}\left((v-\tau)-\frac{1}{m}\right)\right) \\
& -f\left(v, X_{n}\left(v-\frac{1}{m}\right), X_{n}\left((v-\tau)-\frac{1}{m}\right)\right) \\
& +f\left(v, X_{n}\left(v-\frac{1}{m}\right), X_{n}\left((v-\tau)-\frac{1}{m}\right)\right) \\
& -f\left(v, X_{n}\left(v-\frac{1}{n}\right), X_{n}\left((v-\tau)-\frac{1}{n}\right)\right) \|^{2} \mathrm{~d} v \\
\leq & 2 c_{6} \int_{0}^{t} \mathbb{E} \| f\left(v, X_{m}\left(v-\frac{1}{m}\right), X_{m}\left((v-\tau)-\frac{1}{m}\right)\right) \\
& -f\left(v, X_{n}\left(v-\frac{1}{m}\right), X_{n}\left((v-\tau)-\frac{1}{m}\right)\right) \|^{2} \mathrm{~d} v, \\
& +2 c_{6} \int_{0}^{t} \mathbb{E} f\left(v, X_{n}\left(v-\frac{1}{m}\right), X_{n}\left((v-\tau)-\frac{1}{m}\right)\right) \\
& -f\left(v, X_{n}\left(v-\frac{1}{n}\right), X_{n}\left((v-\tau)-\frac{1}{n}\right)\right) \|^{2} \mathrm{~d} v \\
\leq & 2 c_{6} \int_{0}^{t} G\left(v, \mathbb{E}\left\|X_{m}\left(v-\frac{1}{m}\right)-X_{n}\left(v-\frac{1}{m}\right)\right\| \|^{2},\right. \\
& \left.\mathbb{E}\left\|X_{n}\left((v-\tau)-\frac{1}{m}\right)-X_{n}\left((v-\tau)-\frac{1}{n}\right)\right\|^{2}\right) \mathrm{d} v, \\
& \left.\mathbb{E}\left\|X_{m}\left((v-\tau)-\frac{1}{m}\right)-X_{n}\left((v-\tau)-\frac{1}{m}\right)\right\|^{2}\right) \mathrm{d} v \\
& +2 \int_{0}^{t} G\left(v, E\left\|X_{n}\left(v-\frac{1}{m}\right)-X_{n}\left(v-\frac{1}{n}\right)\right\|^{2},\right. \\
&
\end{aligned}
$$


where $c_{6}=\left(2 T^{2 \alpha-1} /(2 \alpha-1) \Gamma^{2}(\alpha)\right)$.

Using Jensen's inequality and Hypothesis 2 again, we acquire

$$
\begin{aligned}
J_{8} \leq & \frac{2}{\Gamma^{2}(\alpha)} \int_{0}^{t}\left(\sup _{0 \leq v<t}(t-v)^{2 \alpha-2}\right) \mathbb{E} \| g\left(v, X_{m}\left(v-\frac{1}{m}\right), X_{m}\left((v-\tau)-\frac{1}{m}\right)\right) \\
& -g\left(v, X_{n}\left(v-\frac{1}{m}\right), X_{n}(v-\tau)-\frac{1}{m}\right) \\
& +g\left(v, X_{n}\left(v-\frac{1}{m}\right), X_{n}\left((v-\tau)-\frac{1}{m}\right)\right) \\
& -g\left(v, X_{n}\left(v-\frac{1}{n}\right), X_{n}\left((v-\tau)-\frac{1}{n}\right)\right) \|^{2} \mathrm{~d} v \\
\leq & \frac{4 \sup _{0 \leq v<t}(t-v)^{2 \alpha-2}}{\Gamma^{2}(\alpha)} \int_{0}^{t} \mathbb{E} \| g\left(v, X_{m}\left(v-\frac{1}{m}\right), X_{m}\left((v-\tau)-\frac{1}{m}\right)\right) \\
& -g\left(v, X_{n}\left(v-\frac{1}{m}\right), X_{n}\left((v-\tau)-\frac{1}{m}\right)\right) \|^{2} \mathrm{~d} v \\
& +\frac{4 \sup _{0 \leq v<t}(t-v)^{2 \alpha-2}}{\Gamma^{2}(\alpha)} \int_{0}^{t} \mathbb{E} \| g\left(v, X_{n}\left(v-\frac{1}{m}\right), X_{n}\left((v-\tau)-\frac{1}{m}\right)\right) \\
& +\frac{4 \sup _{0 \leq v<t}(t-v)^{2 \alpha-2}}{\Gamma^{2}(\alpha)} \int_{0}^{t} G\left(v, \mathbb{E}\left\|X_{n}\left(v-\frac{1}{m}\right)-X_{n}\left(v-\frac{1}{n}\right)\right\|^{2},\right. \\
& \left.\mathbb{E} \| X_{n}\left((v-\tau)-\frac{1}{m}\right)-X_{n}\left(v-\frac{1}{n}\right), X_{n}\left((v-\tau)-\frac{1}{n}\right)\right) \|^{2} \mathrm{~d} v \\
\leq & \frac{4 \sup _{0 \leq v<t}(t-v)^{2 \alpha-2}}{\Gamma^{2}(\alpha)} \int_{0}^{t} G\left(v, \mathbb{E}\left\|X_{m}\left(v-\frac{1}{m}\right)-X_{n}\left(v-\frac{1}{m}\right)\right\|^{2},\right. \\
& \left.\left.\| v-\tau)-\frac{1}{m}\right)-X_{n}\left((v-\tau)-\frac{1}{m}\right) \|^{2}\right) \mathrm{d} v \\
& \\
&
\end{aligned}
$$


In terms of Step 2, we can conclude that

$$
\begin{aligned}
& \mathbb{E}\left(\sup _{0 \leq s \leq t}\left\|X_{m}(s)-X_{n}(s)\right\|^{2}\right) \\
& \leq J_{7}+J_{8} \\
& \leq\left(2 c_{6}+\frac{4 \sup _{0 \leq v<t}(t-v)^{2 \alpha-2}}{\Gamma^{2}(\alpha)}\right) \int_{0}^{t} G\left(v, \mathbb{E}\left\|X_{m}\left(v-\frac{1}{m}\right)-X_{n}\left(v-\frac{1}{m}\right)\right\|^{2},\right. \\
& \left.\mathbb{E}\left\|X_{m}\left((v-\tau)-\frac{1}{m}\right)-X_{n}\left((v-\tau)-\frac{1}{m}\right)\right\|^{2}\right) \mathrm{d} v \\
& +\left(2 c_{6}+\frac{4 \sup _{0 \leq v<t}(t-v)^{2 \alpha-2}}{\Gamma^{2}(\alpha)}\right) \int_{0}^{t} G\left(v, \mathbb{E}\left\|X_{n}\left(v-\frac{1}{m}\right)-X_{n}\left(v-\frac{1}{n}\right)\right\|^{2},\right. \\
& \left.\mathbb{E}\left\|X_{n}\left((v-\tau)-\frac{1}{m}\right)-X_{n}\left((v-\tau)-\frac{1}{n}\right)\right\|^{2}\right) \mathrm{d} v \\
& \leq\left(2 c_{6}+\frac{4 \sup _{0 \leq v<t}(t-v)^{2 \alpha-2}}{\Gamma^{2}(\alpha)}\right) \int_{0}^{t} G\left(v, \mathbb{E}\left(\sup _{0 \leq u_{3} \leq v}\left\|X_{m}\left(u_{3}\right)-X_{n}\left(u_{3}\right)\right\|^{2}\right),\right. \\
& \left.\mathbb{E}\left(\sup _{0 \leq u_{4} \leq v}\left\|X_{m}\left(u_{4}\right)-X_{n}\left(u_{4}\right)\right\|^{2}\right)\right) \mathrm{d} v \\
& +\left(2 c_{6}+\frac{4 \sup _{0 \leq v<t}(t-v)^{2 \alpha-2}}{\Gamma^{2}(\alpha)}\right) \int_{0}^{t} G\left(v, r_{1}\left(\frac{1}{n}-\frac{1}{m}\right)^{2 \alpha-(3 / 2)}+r_{2}\left(\frac{1}{n}-\frac{1}{m}\right)^{2 \alpha-1},\right. \\
& \left.r_{1}\left(\frac{1}{n}-\frac{1}{m}\right)^{2 \alpha-(3 / 2)}+r_{2}\left(\frac{1}{n}-\frac{1}{m}\right)^{2 \alpha-1}\right) \mathrm{d} v \\
& =\left(2 c_{6}+\frac{4 \sup _{0 \leq v<t}(t-v)^{2 \alpha-2}}{\Gamma^{2}(\alpha)}\right) \int_{0}^{t} G\left(v, \mathbb{E}\left(\sup _{0 \leq u \leq v}\left\|X_{m}(u)-X_{n}(u)\right\|^{2}\right)\right) \mathrm{d} v \\
& +\left(2 c_{6}+\frac{4 \sup _{0 \leq v<t}(t-v)^{2 \alpha-2}}{\Gamma^{2}(\alpha)}\right) \int_{0}^{t} G\left(v, r_{1}\left(\frac{1}{n}-\frac{1}{m}\right)^{2 \alpha-(3 / 2)}+r_{2}\left(\frac{1}{n}-\frac{1}{m}\right)^{2 \alpha-1}\right) \mathrm{d} v .
\end{aligned}
$$

Let

$$
Y(t)=\lim _{m, n \longrightarrow \infty} \mathbb{E}\left(\sup _{0 \leq s \leq t}\left\|X_{m}(s)-X_{n}(s)\right\|^{2}\right) .
$$

Then,

$Y(t) \leq\left(2 c_{6}+\frac{4 \sup _{0 \leq v<t}(t-v)^{2 \alpha-2}}{\Gamma^{2}(\alpha)}\right) \int_{0}^{t} G(v, Y(v)) \mathrm{d} v$.
Thus, by Hypothesis 2, we have

$$
Y(t)=\lim _{m, n \longrightarrow \infty} \mathbb{E}\left(\sup _{0 \leq s \leq t}\left\|X_{m}(s)-X_{n}(s)\right\|^{2}\right)=0,
$$

indicating that $\left\{X_{n}(t), n \geq 1\right\}$ is a Cauchy sequence. The Borel-Cantelli lemma makes clear, as $n \longrightarrow \infty, X_{n}(t) \longrightarrow X$ $(t), t \in[0, T]$ holds uniformly. So, if we take the limit of both sides of (16), we get that $X(t)$ is a solution to (1), with the property 


$$
\mathbb{E}\left(\sup _{0 \leq s \leq t}\|X(s)\|^{2}\right)<\infty, \quad 0 \leq t \leq T .
$$

Now, we have proved the existence. The uniqueness of the solutions can be proved in the same way as Step 3. When $t \in[-\tau, 0], X(t)=\Phi(t)$, obviously, there is a unique solution to FSDEs. The proof is complete.

Remark 2. If $G(t, U, V)=\bar{a}(U+V), \bar{a}$ is a constant, then Hypothesis 2 and Hypothesis 3 are equivalent to Hypothesis 1. Therefore, under Hypothesis 1 and some proper conditions, there will exist a unique solution $X(t)$ to FSDEs (1).

\section{Ulam-Hyers Stability Analysis of FSDEs}

We are going to research the solution $X(t), t \in[0, T]$ of system (1) is Ulam-Hyers stable and prove the stability theory of solutions to FSDEs (1) with Lipschitz and non-Lipschitz coefficients in this section.
Theorem 2. Assume that Hypothesis 1 holds and $12 l^{2} T^{2 \alpha-(1 / 2)}<(4 \alpha-3)^{(1 / 2)} \Gamma^{2}(\alpha),(3 / 4)<\alpha<1$. The FSDE (1) is Ulam-Hyers which is stable at $[0, T]$.

Proof. From Definition 3 and Remark 1, we know

$$
\begin{aligned}
Z(t)= & \Phi_{0}+\frac{1}{\Gamma(\alpha)} \int_{0}^{t}(t-v)^{\alpha-1} f(v, Z(v), Z(v-\tau)) \mathrm{d} v \\
& +\frac{1}{\Gamma(\alpha)} \int_{0}^{t}(t-v)^{\alpha-1} g(v, Z(v), Z(v-\tau)) \mathrm{d} W(v) \\
& +\frac{1}{\Gamma(\alpha)} \int_{0}^{t}(t-v)^{\alpha-1} h(v) \mathrm{d} v .
\end{aligned}
$$

According to Definition 3 and equation (45), we have

$$
\begin{aligned}
Z(t) & -X(t) \\
= & \frac{1}{\Gamma(\alpha)} \int_{0}^{t}(t-v)^{\alpha-1}(f(v,(v), Z(v-\tau))-f(v, X(v), X(v-\tau))) \mathrm{d} v \\
& +\frac{1}{\Gamma(\alpha)} \int_{0}^{t}(t-v)^{\alpha-1}(g(v, Z(v), Z(v-\tau))-g(v, Z(v), Z(v-\tau))) \mathrm{d} W(v) \\
& +\frac{1}{\Gamma(\alpha)} \int_{0}^{t}(t-v)^{\alpha-1} h(v) \mathrm{d} v,
\end{aligned}
$$

and then using Jensen's inequality, we obtain

$$
\begin{aligned}
& \mathbb{E}\left(\sup _{0 \leq t \leq T}\|Z(t)-X(t)\|^{2}\right) \\
& \leq \frac{3}{\Gamma^{2}(\alpha)} \mathbb{E}\left(\sup _{0 \leq t \leq T} \| \int_{0}^{t}(t-v)^{\alpha-1}(f(v, Z(v), Z(v-\tau))\right. \\
& \left.-f v, X(v), X(v-\tau))) \mathrm{d} v \|^{2}\right) \\
& +\frac{3}{\Gamma^{2}(\alpha)} \mathbb{E}\left(\sup _{0 \leq t \leq T} \| \int_{0}^{t}(t-v)^{\alpha-1}(g(v, Z(v), Z(v-\tau))\right. \\
& \left.-g(v, X(v), X(v-\tau))) \mathrm{d} W(v) \|^{2}\right) \\
& +\frac{3}{\Gamma^{2}(\alpha)} \mathbb{E}\left(\sup _{0 \leq t \leq T}\left\|\int_{0}^{t}(t-v)^{\alpha-1} h(v) \mathrm{d} v\right\|^{2}\right)=I_{1}+I_{2}+I_{3} .
\end{aligned}
$$


Now, we use Hölder's inequality and Hypothesis 1, and one can obtain

$$
\begin{aligned}
I_{1} \leq & \frac{3}{\Gamma^{2}(\alpha)}\left(\sup _{0 \leq t \leq T} \int_{0}^{t}(t-v)^{2 \alpha-2} \mathrm{~d} v\right) \\
& \cdot \mathbb{E} \int_{0}^{T}\|f(v, Z(v), Z(v-\tau))-f(v, X(v), X(v-\tau))\|^{2} \mathrm{~d} v \\
\leq & \frac{3 l^{2} T^{2 \alpha-1}}{(2 \alpha-1) \Gamma^{2}(\alpha)} \mathbb{E} \int_{0}^{T}(\|Z(v)-X(v)\|+\|Z(v-\tau)+X(v-\tau)\|)^{2} \mathrm{~d} v \\
\leq & \frac{6 l^{2} T^{2 \alpha-1}}{(2 \alpha-1) \Gamma^{2}(\alpha)} \int_{0}^{T}\left(\mathbb{E}\|Z(v)-X(v)\|^{2}+\mathbb{E}\|Z(v-\tau)-X(v-\tau)\|^{2}\right) \mathrm{d} v \\
= & b_{1} T^{2 \alpha-1} \int_{0}^{T} \mathbb{E}\left(\|Z(v)-X(v)\|^{2}\right) \mathrm{d} v \\
& +b_{1} T^{2 \alpha-1} \int_{0}^{T} \mathbb{E}\left(\|Z(v-\tau)-X(v-\tau)\|^{2}\right) \mathrm{d} v,
\end{aligned}
$$

where $b_{1}=\left(6 l^{2} /(2 \alpha-1) \Gamma^{2}(\alpha)\right)$.

Then, by Itô isometry and Hölder's inequality, we obtain

$$
\begin{aligned}
I_{2} \leq & \frac{3}{\Gamma^{2}(\alpha)} \mathbb{E}\left\|\int_{0}^{T}(T-v)^{\alpha-1}(g(v, Z(v), Z(v-\tau))-g(v, X(v), X(v-\tau))) \mathrm{d} W(v)\right\|^{2} \\
& =\frac{3}{\Gamma^{2}(\alpha)} \mathbb{E}\left(\int_{0}^{T}\left\|(T-v)^{\alpha-1}(g(v, Z(v), Z(v-\tau))-g(v, X(v), X(v-\tau)))\right\|^{2} \mathrm{~d} v\right) \\
& \leq \frac{3}{\Gamma^{2}(\alpha)}\left(\int_{0}^{T}(T-v)^{4 \alpha-4} \mathrm{~d} v\right)^{(1 / 2)} \\
& \cdot \mathbb{E}\left(\int_{0}^{T}\|g(v, Z(v), Z(v-\tau))-g(v, X(v), X(v-\tau))\|^{4} \mathrm{~d} v\right)^{(1 / 2)} \\
& \frac{3 T^{2 \alpha-(3 / 2)}}{(4 \alpha-3)^{(1 / 2)} \Gamma^{2}(\alpha)} \mathbb{E}\left(\int_{0}^{T}\|g(v, Z(v), Z(v-\tau))-g(v, X(v), X(v-\tau))\|^{4} \mathrm{~d} v\right)^{(1 / 2)} \\
& =b_{2} T^{2 \alpha-(3 / 2)} \mathbb{E}\left(\int_{0}^{T}\|g(v, Z(v), Z(v-\tau))-g(v, X(v), X(v-\tau))\|^{4} \mathrm{~d} v\right)^{(1 / 2)},
\end{aligned}
$$

where $b_{2}=\left(3 /(4 \alpha-3)^{(1 / 2)} \Gamma^{2}(\alpha)\right)$. Since $\| g(v, Z(v), Z(v-$ $\tau))-g(v, X(v), X(v-\tau)) \|^{4}$ is a continuous function on $[0, T]$, according to the mean value theorem of integrals, there exists $\hat{y} \in[0, T]$, such that

$$
\begin{aligned}
& \int_{0}^{T} \| g(v, Z(v), Z(v-\tau))-g\left(v, X(v), X(v-\tau) \|^{4} \mathrm{~d} v\right. \\
& =T \| g(\widehat{y}, Z(\widehat{y}), Z(\widehat{y}-\tau))-g\left(\widehat{y}, X(\widehat{y}), X(\widehat{y}-\tau) \|^{4} .\right.
\end{aligned}
$$


By Hypothesis 1 and Jensen's inequality, we obtain

$$
\begin{aligned}
I_{2} & \leq b_{2} T^{2 \alpha-(1 / 2)} \mathbb{E} \| g\left((\hat{y}, Z(\widehat{y}), Z(\widehat{y}-\tau))-g(\hat{y}, X(\widehat{y}), X(\widehat{y}-\tau)) \|^{2}\right. \\
& \leq 2 l^{2} b_{2} T^{2 \alpha-(1 / 2)}\left(\mathbb{E}\|Z(\widehat{y})-X(\widehat{y})\|^{2}+\mathbb{E}\|Z(\widehat{y}-\tau)-X(\widehat{y}-\tau)\|^{2}\right) .
\end{aligned}
$$

Finally, we use Cauchy-Schwarz inequality and Remark 1 to yield

$$
\begin{aligned}
I_{3} & \leq \frac{3}{\Gamma^{2}(\alpha)} \mathbb{E}\left[\sup _{0 \leq t \leq T}\left(\int_{0}^{t}(t-v)^{2 \alpha-2} \mathrm{~d} v \cdot \int_{0}^{t}\|h(v)\|^{2} \mathrm{~d} v\right)\right] \\
& \leq \frac{3 T^{2 \alpha-1}}{(2 \alpha-1) \Gamma^{2}(\alpha)}\left(\int_{0}^{T} \mathbb{E}\left(\sup _{0 \leq v \leq T}\|h(v)\|^{2}\right)\right) \mathrm{d} v \\
& \leq \frac{3 T^{2 \alpha} \varepsilon}{(2 \alpha-1) \Gamma^{2}(\alpha)} \\
& =b_{3} T^{2 \alpha} \varepsilon,
\end{aligned}
$$

where $b_{3}=\left(3 /(2 \alpha-1) \Gamma^{2}(\alpha)\right)$.

Hence, we obtain

$$
\begin{aligned}
& \mathbb{E}\left(\sup _{0 \leq t \leq T}\|Z(t)-X(t)\|^{2}\right) \\
& \leq b_{1} T^{2 \alpha-1}\left(\int_{0}^{T} \mathbb{E}\left(\|Z(v)-X(v)\|^{2}\right) \mathrm{d} v+\int_{0}^{T} \mathbb{E}\left(\|Z(v-\tau)-X(v-\tau)\|^{2}\right) \mathrm{d} v\right) \\
& \quad+2 l^{2} b_{2} T^{2 \alpha-(1 / 2)}\left(\mathbb{E}\|Z(\widehat{y})-X(\widehat{y})\|^{2}+\mathbb{E}\|Z(\widehat{y}-\tau)-X(\widehat{y}-\tau)\|^{2}\right)+b_{3} T^{2 \alpha} \varepsilon \\
& \leq b_{1} T^{2 \alpha-1} \int_{0}^{T} \mathbb{E}\left(\sup _{0 \leq s_{1} \leq v}\left\|Z\left(s_{1}\right)-X\left(s_{1}\right)\right\|^{2}\right) \mathrm{d} v \\
& \quad+b_{1} T^{2 \alpha-1} \int_{0}^{T} \mathbb{E}\left(\sup _{0 \leq s_{1} \leq v}\left\|Z\left(s_{1}-\tau\right)-X\left(s_{1}-\tau\right)\right\|^{2}\right) \mathrm{d} v \\
& \quad+2 l^{2} b_{2} T^{2 \alpha-(1 / 2)} \mathbb{E}\left(\sup _{0 \leq \hat{y}_{1} \leq \hat{y}}\left\|Z\left(\widehat{y}_{1}\right)-X\left(\widehat{y}_{1}\right)\right\|^{2}\right) \\
& \quad+2 l^{2} b_{2} T^{2 \alpha-(1 / 2)} \mathbb{E}\left(\sup _{0 \leq \widehat{y}_{1} \leq \hat{y}}\left\|Z\left(\widehat{y}_{1}-\tau\right)-X\left(\widehat{y}_{1}-\tau\right)\right\|^{2}\right)+b_{3} T^{2 \alpha} \varepsilon .
\end{aligned}
$$

Different from the approach of dealing with the delay in $[18,39,40]$, we obtain

$$
\begin{aligned}
V(T) & =\mathbb{E}\left(\sup _{0 \leq t \leq T}\|Z(t)-X(t)\|^{2}\right), \\
\mathbb{E}\left(\sup _{-\tau \leq t \leq 0}\|Z(t)-X(t)\|^{2}\right) & =0,
\end{aligned}
$$

and then, we acquire

$$
\mathbb{E}\left(\sup _{0 \leq s_{1} \leq v}\left\|Z\left(s_{1}-\tau\right)-X\left(s_{1}-\tau\right)\right\|^{2}\right)=V(v-\tau) .
$$

Hence,

$$
\begin{aligned}
V(T) \leq & b_{1} T^{2 \alpha-1}\left(\int_{0}^{T} V(v) \mathrm{d} v+\int_{0}^{T} V(v-\tau) \mathrm{d} v\right) \\
& +2 l^{2} b_{2} T^{2 \alpha-(1 / 2)}(V(\widehat{y})+V(\widehat{y}-\tau))+b_{3} T^{2 \alpha} \varepsilon .
\end{aligned}
$$

Let us set $U(T)=\sup V(\theta)$, then $V(v) \leq U(v)$, and $V(v-\tau) \leq U(v)$. Thus, $\theta \in[-\tau, T]$

$$
V(T) \leq 2 b_{1} T^{2 \alpha-1} \int_{0}^{T} U(v) \mathrm{d} v+4 l^{2} b_{2} T^{2 \alpha-(1 / 2)} U(\widehat{y})+b_{3} T^{2 \alpha} \varepsilon .
$$


For $\forall \theta \in[0, T]$, we obtain

$$
\begin{aligned}
V(\theta) & \leq 2 b_{1} \theta^{2 \alpha-1} \int_{0}^{\theta} U(v) \mathrm{d} v+4 l^{2} b_{2} \theta^{2 \alpha-(1 / 2)} U(\widehat{y})+b_{3} \theta^{2 \alpha} \varepsilon \\
& \leq 2 b_{1} T^{2 \alpha-1} \int_{0}^{T} U(v) \mathrm{d} v+4 l^{2} b_{2} T^{2 \alpha-(1 / 2)} U(\widehat{y})+b_{3} T^{2 \alpha} \varepsilon .
\end{aligned}
$$

Then, we can obtain

$$
\begin{aligned}
U(T) & =\sup _{\theta \in[-\tau, T]} V(\theta) \\
& \leq \max \left\{\sup _{\theta \in[-\tau, 0]} V(\theta), \sup _{\theta \in[0, T]} V(\theta)\right\} \\
& \leq 2 b_{1} T^{2 \alpha-1} \int_{0}^{T} U(v) \mathrm{d} v+4 l^{2} b_{2} T^{2 \alpha-(1 / 2)} U(T)+b_{3} T^{2 \alpha} \varepsilon .
\end{aligned}
$$

Then,

$$
U(T) \leq \frac{2 b_{1} T^{2 \alpha-1}}{1-4 l^{2} b_{2} T^{2 \alpha-(1 / 2)}} \int_{0}^{T} U(v) \mathrm{d} v+\frac{b_{3} T^{2 \alpha} \varepsilon}{1-4 l^{2} b_{2} T^{2 \alpha-(1 / 2)}}
$$

Using Gronwall's inequality, we obtain

$$
U(T) \leq \frac{b_{3} T^{2 \alpha} \varepsilon}{1-4 l^{2} b_{2} T^{2 \alpha-(1 / 2)}} e^{\left(2 b_{1} T^{\left.2 \alpha / 1-4 l^{2} b_{2} T^{2 \alpha-(1 / 2)}\right)} .\right.}
$$

Therefore,

$$
\begin{aligned}
\mathbb{E}\left(\sup _{0 \leq t \leq T}\|Z(t)-X(t)\|^{2}\right) \leq & \frac{b_{3} T^{2 \alpha} \varepsilon}{1-4 l^{2} b_{2} T^{2 \alpha-(1 / 2)}} \\
& \cdot e^{\left(2 b_{1} T^{2 \alpha} / 1-4 l^{2} b_{2} T^{2 \alpha-(1 / 2)}\right)},
\end{aligned}
$$

consequently, $\forall \varepsilon>0 ; \quad$ there exists $\delta=\left(b_{3} T^{2 \alpha} /\right.$
$\left.1-4 l^{2} b_{2} T^{2 \alpha-(1 / 2)}\right) e^{\left(2 b_{1} T^{2 \alpha} / 1-4 l^{2} b_{2} T^{2 \alpha-(1 / 2)}\right)}$, such that

$$
\mathbb{E}\left(\sup _{0 \leq t \leq T}\|Z(t)-X(t)\|^{2}\right) \leq \varepsilon \delta .
$$

Therefore, this theorem is proved.

Theorem 3. Assume that Hypothesis 2 and Hypothesis 3 hold, $6 \tilde{k} T^{2 \alpha-(1 / 2)}<(4 \alpha-3)^{(1 / 2)} \Gamma^{2}(\alpha), \quad \tilde{k}=\max \left\{\sup _{0 \leq t \leq T} b(t)\right.$, $\left.\sup _{0 \leq t \leq T} q(t)\right\}$, and there exists a constant $\gamma$ satisfying $\left(3(4 \alpha-3)^{(1 / 2)} T^{2 \alpha}+3(2 \alpha-1) \quad T^{2 \alpha-(1 / 2)} /(2 \alpha-1) \quad(4 \alpha-\right.$ $\left.3)^{(1 / 2)} \Gamma^{2}(\alpha)-6(2 \alpha-1) \tilde{k} T^{2 \alpha-(1 / 2)}\right) \sup _{0 \leq t \leq T} a(t) \leq \gamma \mathcal{\varepsilon}, \quad(3 / 4)<$ $\alpha<1$. The FSDE (1) is Ulam-Hyers which is stable at $[0, T]$.

Proof. From inequality (48), we obtain

$$
\begin{aligned}
& \mathbb{E}\left(\sup _{0 \leq t \leq T}\|Z(t)-X(t)\|^{2}\right) \\
& \leq \frac{3}{\Gamma^{2}(\alpha)} \mathbb{E}\left(\sup _{0 \leq t \leq T}\left\|\int_{0}^{t}(t-v)^{\alpha-1}(f(v, Z(v), Z(v-\tau))-f(v, X(v), X(v-\tau))) \mathrm{d} v\right\|^{2}\right) \\
& \quad+\frac{3}{\Gamma^{2}(\alpha)} \mathbb{E}\left(\sup _{0 \leq t \leq T}\left\|\int_{0}^{t}(t-v)^{\alpha-1}(g(v, Z(v), Z(v-\tau))-g(v, X(v), X(v-\tau))) \mathrm{d} W(v)\right\|^{2}\right) \\
& \quad+\frac{3}{\Gamma^{2}(\alpha)} \mathbb{E}\left(\sup _{0 \leq t \leq T}\left\|\int_{0}^{t}(t-v)^{\alpha-1} h(v) \mathrm{d} v\right\|^{2}\right) \\
& =I_{1}+I_{2}+I_{3} .
\end{aligned}
$$

Taking inequality (48) and Hypothesis 2 into account to achieve,

$$
\begin{aligned}
I_{1} & \leq \frac{3 T^{2 \alpha-1}}{(2 \alpha-1) \Gamma^{2}(\alpha)} \mathbb{E}\left(\int_{0}^{T}\|f(v, Z(v), Z(v-\tau))-f(v, X(v), X(v-\tau))\|^{2} \mathrm{~d} v\right) \\
& \leq b_{4} T^{2 \alpha-1} \int_{0}^{T} \mathbb{E} G\left(v,\|Z(v)-X(v)\|^{2},\|Z(v-\tau)-X(v-\tau)\|^{2}\right) \mathrm{d} v,
\end{aligned}
$$

where $b_{4}=\left(3 /(2 \alpha-1) \Gamma^{2}(\alpha)\right)$. 
By inequalities (50)-(52) and Hypothesis 2, one can obtain

$$
\begin{aligned}
I_{2} & \leq b_{2} T^{2 \alpha-(1 / 2)} \mathbb{E}\|g(\hat{y}, Z(\hat{y}), Z(\hat{y}-\tau))-g(\hat{y}, X(\hat{y}), X(\hat{y}-\tau))\|^{2} \\
& \leq b_{2} T^{2 \alpha-(1 / 2)} \mathbb{E} G\left(\hat{y},\|Z(\hat{y})-X(\hat{y})\|^{2},\|Z(\hat{y}-\tau)-X(\hat{y}-\tau)\|^{2}\right) .
\end{aligned}
$$

Using Hypothesis 3, it is immediate to obtain

$$
\begin{aligned}
I_{1}+ & I_{2} \\
\leq & b_{4} T^{2 \alpha-1} \int_{0}^{T} \mathbb{E} G\left(v, Z(v)-X(v)\left\|^{2},\right\| Z(v-\tau)-X(v-\tau) \|^{2}\right) \mathrm{d} v \\
& +b_{2} T^{2 \alpha-(1 / 2)} \mathbb{E} G\left(\hat{y},\|Z(\widehat{y})-X(\hat{y})\|^{2},\|Z(\hat{y}-\tau)-X(\hat{y}-\tau)\|^{2}\right) \\
\leq & b_{4} T^{2 \alpha-1} \int_{0}^{T} \mathbb{E}\left(a(v)+b(v)\|Z(v)-X(v)\|^{2}+q(v)\|Z(v-\tau)-X(v-\tau)\|^{2}\right) \mathrm{d} v \\
& +b_{2} T^{2 \alpha-(1 / 2)} \mathbb{E}\left(a(\hat{y})+b(\hat{y})\|Z(\hat{y})-X(\widehat{y})\|^{2}+q(\hat{y})\|Z(\hat{y}-\tau)-X(\hat{y}-\tau)\|^{2}\right) \\
\leq & \left(b_{4} T^{2 \alpha}+b_{2} T^{2 \alpha-(1 / 2)}\right) \sup _{0 \leq v \leq T} a(v) \\
& +b_{4} \widetilde{k} T^{2 \alpha-1} \int_{0}^{T}\left(\mathbb{E}\|Z(v)-X(v)\|^{2}+\mathbb{E}\|Z(v-\tau)-X(v-\tau)\|^{2}\right) \mathrm{d} v \\
& +b_{2} \widetilde{k} T^{2 \alpha-(1 / 2)}\left(\mathbb{E}\|Z(\hat{y})-X(\hat{y})\|^{2}+\mathbb{E}\|Z(\hat{y}-\tau)-X(\hat{y}-\tau)\|^{2}\right) .
\end{aligned}
$$

Now, through inequality (52), we have

$$
I_{3} \leq b_{3} T^{2 \alpha} \varepsilon
$$

Then,

$$
\begin{aligned}
& \mathbb{E}\left(\sup _{0 \leq t \leq T}\|Z(t)-X(t)\|^{2}\right) \\
& \leq\left(b_{4} T^{2 \alpha}+b_{2} T^{2 \alpha-(1 / 2)}\right) \sup _{0 \leq v \leq T} a(v) \\
& \quad+b_{4} \widetilde{k} T^{2 \alpha-1} \int_{0}^{T} \mathbb{E}\left(\sup _{0 \leq s_{1} \leq v}\left\|Z\left(s_{1}\right)-X\left(s_{1}\right)\right\|^{2}\right) \mathrm{d} v \\
& +b_{4} \widetilde{k} T^{2 \alpha-1} \int_{0}^{T} \mathbb{E}\left(\sup _{0 \leq s_{1} \leq v}\left\|Z\left(s_{1}-\tau\right)-X\left(s_{1}-\tau\right)\right\|^{2}\right) \mathrm{d} v \\
& +b_{2} \widetilde{k} T^{2 \alpha-(1 / 2)} \mathbb{E}\left(\sup _{0 \leq s_{2} \leq \widehat{y}}\left\|Z\left(s_{2}\right)-X\left(s_{2}\right)\right\|^{2}\right) \\
& +b_{2} \widetilde{k} T^{2 \alpha-(1 / 2)} \mathbb{E}\left(\sup _{0 \leq s_{2} \leq \widehat{y}}\left\|Z\left(s_{2}-\tau\right)-X\left(s_{2}-\tau\right)\right\|^{2}\right) \\
& \quad+b_{3} T^{2 \alpha}{ }_{\varepsilon} .
\end{aligned}
$$

Let us set $V(T)=\mathbb{E}\left(\sup _{0 \leq t \leq T}\|Z(t)-X(t)\|^{2}\right)$ and $\mathbb{E}\left(\sup \sup \|Z(t)-X(t)\|^{2}\right)=0$, we can obtain

$$
\mathbb{E}\left(\sup _{0 \leq s_{1} \leq v}\left\|Z\left(s_{1}-\tau\right)-X\left(s_{1}-\tau\right)\right\|^{2}\right)=V(v-\tau) .
$$

Indeed, we can conclude that

$$
\begin{aligned}
V(T) \leq & b_{4} \tilde{k} T^{2 \alpha-1}\left(\int_{0}^{T} V(v) \mathrm{d} v+\int_{0}^{T} V(v-\tau) \mathrm{d} v\right) \\
& +b_{2} \tilde{k} T^{2 \alpha-(1 / 2)}(V(\widehat{y})+V(\widehat{y}-\tau)) \\
& +\left(b_{4} T^{2 \alpha}+b_{2} T^{2 \alpha-(1 / 2)}\right) \sup _{0 \leq v \leq T} a(v)+b_{3} T^{2 \alpha} \varepsilon .
\end{aligned}
$$

Letting $U(T)=\sup _{\theta \in[-\tau, T]} V(\theta)$, then $V(v) \leq U(v)$ and $V(v-\tau) \leq U(v)$. Thus,

$$
\begin{aligned}
V(T) \leq & 2 b_{4} \tilde{k} T^{2 \alpha-1} \int_{0}^{T} U+2 b_{2} \tilde{k} T^{2 \alpha-(1 / 2)} U(\widehat{y}) \\
& +\left(b_{4} T^{2 \alpha}+b_{2} T^{2 \alpha-(1 / 2)}\right) \sup _{0 \leq v \leq T} a(v)+b_{3} T^{2 \alpha} \varepsilon .
\end{aligned}
$$

For $\forall \theta \in[0, T]$, we get that

$$
\begin{aligned}
V(\theta) \leq & 2 b_{4} \widetilde{k} \theta^{2 \alpha-1} \int_{0}^{\theta} U(v) \mathrm{d} v+2 b_{2} \widetilde{k} \theta^{2 \alpha-(1 / 2)} U(\widehat{y}) \\
& +\left(b_{4} \theta^{2 \alpha}+b_{2} \theta^{2 \alpha-(1 / 2)}\right) \sup _{0 \leq v \leq \theta} a(v)+b_{3} \theta^{2 \alpha} \varepsilon \\
\leq & 2 b_{4} \tilde{k} T^{2 \alpha-1} \int_{0}^{T} U(v) \mathrm{d} v+2 b_{2} \tilde{k} T^{2 \alpha-(1 / 2)} U(T) \\
& +\left(b_{4} T^{2 \alpha}+b_{2} T^{2 \alpha-(1 / 2)}\right) \sup _{0 \leq v \leq T} a(v)+b_{3} T^{2 \alpha} \varepsilon
\end{aligned}
$$


Moreover, we have

$$
\begin{aligned}
U(T)= & \sup _{\theta \in[-\tau, T]} V(\theta) \\
\leq & \max \left\{\sup _{\theta \in[-\tau, 0]} V(\theta), \sup _{\theta \in[0, T]} V(\theta)\right\} \\
\leq & 2 b_{4} \tilde{k} T^{2 \alpha-1} \int_{0}^{T} U(v) \mathrm{d} v+2 b_{2} \tilde{k} T^{2 \alpha-(1 / 2)} U(T) \\
& +\left(b_{4} T^{2 \alpha}+b_{2} T^{2 \alpha-(1 / 2)}\right) \sup _{0 \leq v \leq T} a(v)+b_{3} T^{2 \alpha} \varepsilon .
\end{aligned}
$$

Furthermore,

$$
\begin{aligned}
U(T) \leq & \frac{2 b_{4} \tilde{k} T^{2 \alpha-1}}{1-2 b_{2} \tilde{k} T^{2 \alpha-(1 / 2)}} \int_{0}^{T} U(v) \mathrm{d} v \\
& +\frac{b_{4} T^{2 \alpha}+b_{2} T^{2 \alpha-(1 / 2)}}{1-2 b_{2} \tilde{k} T^{2 \alpha-(1 / 2)}} \sup _{0 \leq \nu \leq T} a(v)+\frac{b_{3} T^{2 \alpha} \varepsilon}{1-2 b_{2} \tilde{k} T^{2 \alpha-(1 / 2)}} .
\end{aligned}
$$

$$
\begin{aligned}
& \mathbb{E}\left(\sup _{0 \leq t \leq T}\|Z(t)-X(t)\|^{2}\right) \\
& \quad \leq\left(\frac{b_{4} T^{2 \alpha}+b_{2} T^{2 \alpha-(1 / 2)}}{1-2 b_{2} \tilde{k} T^{2 \alpha-(1 / 2)}} \sup _{0 \leq \nu \leq T} a(v)+\frac{b_{3} T^{2 \alpha} \varepsilon}{1-2 b_{2} \tilde{k} T^{2 \alpha-(1 / 2)}}\right) e^{\left(2 b_{4} \tilde{k} T^{2 \alpha / 1-2 b_{2}} \tilde{k} T^{2 \alpha-(1 / 2)}\right)} \\
& \quad \leq\left(\gamma+\frac{b_{3} T^{2 \alpha}}{1-2 b_{2} \tilde{k} T^{2 \alpha-(1 / 2)}}\right) \varepsilon e^{\left(2 b_{4} \tilde{k} T^{2 \alpha+1} / 1-2 b_{2} \tilde{k} T^{2 \alpha-(1 / 2)}\right)},
\end{aligned}
$$

which implies that there exists $\delta=\left(\gamma+\left(b_{3} T^{2 \alpha} / 1-2 b_{2} \widetilde{k}\right.\right.$ $\left.\left.\left.T^{2 \alpha-(1 / 2)}\right)\right) e^{\left(2 b_{4} \tilde{k} T^{2 \alpha} / 1-2 b_{2}\right.} \tilde{k} T^{2 \alpha-(1 / 2)}\right)>0, \forall \varepsilon>0$, satisfying

$$
\mathbb{E}\left(\sup _{0 \leq t \leq T}\|Z(t)-X(t)\|^{2}\right) \leq \varepsilon \delta .
$$

This completes the proof.

\section{Examples}

Example 1. Consider the Ulam-Hyers stability and existence and uniqueness of the solution to the following equation:

$$
\begin{aligned}
{ }^{C} D_{0^{+}}^{(4 / 5)} X(t)= & \frac{1}{15} \sin X(t)+\frac{2}{23} \cos X(t-\tau) \\
& +\left[\frac{\Gamma(1 / 2)}{24 \pi} \cos ^{2} X(t)+\frac{\ln (3 / 2)}{\pi e^{4}} X(t-\tau)\right] \frac{\mathrm{d} W(t)}{\mathrm{d} t},
\end{aligned}
$$

where $\alpha=(4 / 5), \quad t \in[0,8]$, and uniformly continuous functions 


$$
\begin{aligned}
& f(t, X(t), X(t-\tau))=\frac{1}{15} \sin X(t)+\frac{2}{23} \cos X(t-\tau), \\
& g(t, X(t), X(t-\tau))=\frac{\Gamma(1 / 2)}{24 \pi} \cos ^{2} X(t)+\frac{\ln (3 / 2)}{\pi e^{4}} X(t-\tau) .
\end{aligned}
$$

$$
\begin{aligned}
& \| f(t, Z(t), Z(t-\tau))-f(t, X(t), X(t-\tau) \| \\
& =\left\|\frac{1}{15} \sin Z(t)+\frac{2}{23} \cos Z(t-\tau)-\frac{1}{15} \sin X(t)-\frac{2}{23} \cos X(t-\tau)\right\| \\
& \leq \frac{1}{15}\|\sin Z(t)-\sin X(t)\|+\frac{2}{23}\|\cos Z(t-\tau)-\cos X(t-\tau)\| \\
& =\frac{1}{15}\left\|2 \cos \frac{Z(t)+X(t)}{2} \sin \frac{Z(t)-X(t)}{2}\right\| \\
& +\frac{2}{23}\left\|-2 \sin \frac{Z(t-\tau)+X(t-\tau)}{2} \sin \frac{Z(t-\tau)-X(t-\tau)}{2}\right\| \\
& \leq \frac{1}{15}\left\|2 \sin \frac{Z(t)-X(t)}{2}\right\|+\frac{2}{23}\left\|2 \sin \frac{Z(t-\tau)-X(t-\tau)}{2}\right\| \\
& \leq \frac{1}{15}\|Z(t)-X(t)\|+\frac{2}{23}\|Z(t-\tau)-X(t-\tau)\| \\
& \leq \frac{2}{23}(\|Z(t)-X(t)\|+\|Z(t-\tau)-X(t-\tau)\|), \\
& \|g(t, Z(t), Z(t-\tau))-g(t, X(t), X(t-\tau))\| \\
& =\left\|\frac{\Gamma(1 / 2)}{24 \pi} \cos ^{2} Z(t)+\frac{\ln (3 / 2)}{\pi e^{4}} Z(t-\tau)-\frac{\Gamma(1 / 2)}{24 \pi} \cos ^{2} X(t)-\frac{\ln (3 / 2)}{\pi e^{4}} X(t-\tau)\right\| \\
& \leq \frac{\Gamma(1 / 2)}{24 \pi}\left\|\cos ^{2} Z(t)-\cos ^{2} X(t)\right\|+\frac{\ln (3 / 2)}{\pi e^{4}}\|Z(t-\tau)-X(\tau-\tau)\| \\
& =\frac{\Gamma(1 / 2)}{24 \pi}\|[\cos Z(t)+\cos X(t)][\cos Z(t)-\cos X(t)]\|+\frac{\ln (3 / 2)}{\pi e^{4}}\|Z(t-\tau)-X(t-\tau)\| \\
& \leq \frac{\Gamma(1 / 2)}{12 \pi}\|\cos Z(t)-\cos X(t)\|+\frac{\ln (3 / 2)}{\pi e^{4}}\|Z(t-\tau)-X(t-\tau)\| \\
& \leq \frac{2}{23}(\|Z(t)-X(t)\|+\|Z(t-\tau)-X(t-\tau)\|)
\end{aligned}
$$

$f(t, X(t), X(t-\tau)$ and $g(t, X(t), X(t-\tau)$ satisfy Hypothesis $1, \quad 3^{(3 / 2)} c T^{2 \alpha-1}=3^{(3 / 2)} \times 2 \times(2 / 23)^{2} \times 5^{(3 / 5)} \approx 0.09<(\alpha-(3 /$ 4) $)^{(1 / 2)} \Gamma^{2}(\alpha)=\sqrt{(1 / 20)} \times \Gamma^{2}(3 / 4) \approx 0.30$ and $12 l^{2} T^{2 \alpha-(1 / 2)}$ $=12 \times(2 / 23)^{2} \times(5)^{(11 / 10)} \approx 0.54<(4 \alpha-3)^{(1 / 2)} \Gamma^{2}(\alpha)=\sqrt{(1 / 5)} \Gamma^{2}$ $(4 / 5) \approx 0.60$. Therefore, according to Remark 2 and Theorem 2 , we can see that there is a unique solution to equation (80) and the solution is Ulam-Hyers stable.
Now, a numerical simulation will be carried out to find the solution of (79) is Ulam-Hyers stable, and we can see it in Figure 1.

Example 2. Consider the Ulam-Hyers stability of the following especial FSDEs with time delays: 


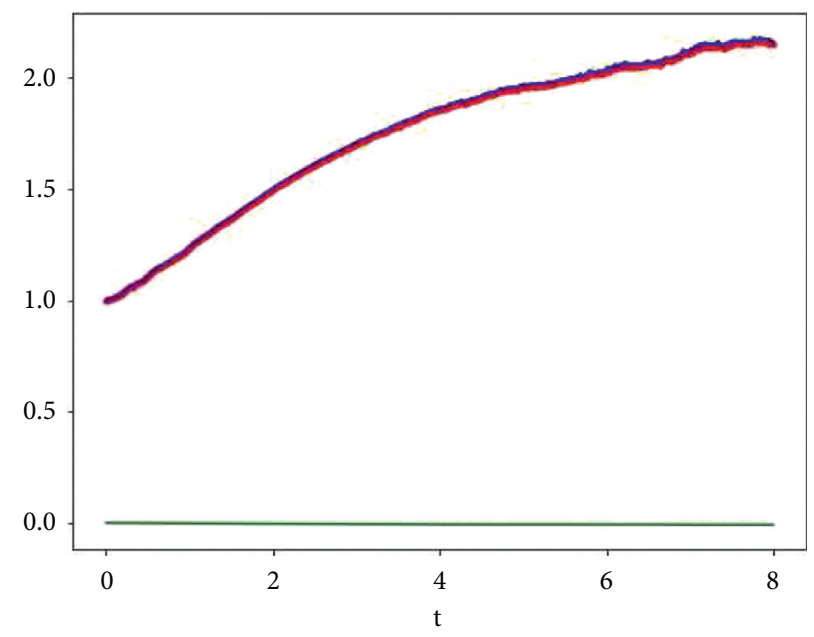

- $X(t)$

$Z(t)$

Er

Figure 1: $X(0)=1, h(t)=\sqrt{\varepsilon}, \mathbb{E}\left(\sup _{0 \leq t \leq 8}\|h(t)\|^{2}\right)=\mathbb{E}\left(\sup _{0 \leq t \leq 8}\|\sqrt{\varepsilon}\|^{2}\right)=\varepsilon, \varepsilon=0.001$.

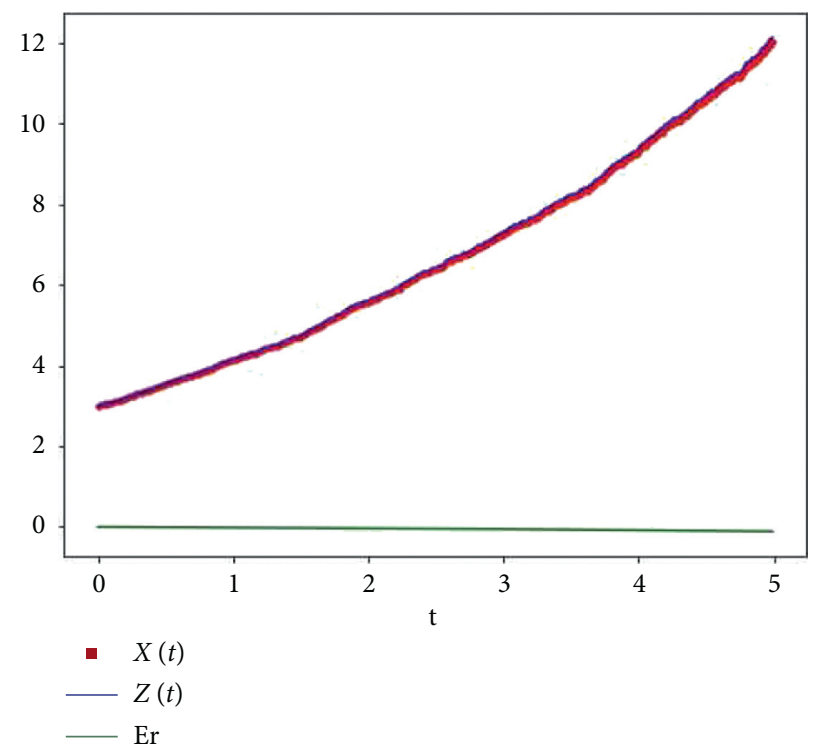

FigURE 2: $X(0)=3, n=12, \widetilde{\varepsilon}=(\sqrt{2} / 9), h(t)=(\sqrt{\varepsilon} / 2), \mathbb{E}\left(\sup _{0 \leq t \leq 2}\|h(t)\|^{2}\right)=\mathbb{E}\left(\sup _{0 \leq t \leq 2}\|\sqrt{\varepsilon} / 2\|^{2}\right)=(\varepsilon / 4)<\varepsilon, \varepsilon=0.007$.

$$
{ }^{C} D_{0^{+}}^{(5 / 6)} X(t)=\frac{1}{n} X(t)+\frac{\widetilde{\varepsilon}}{\sqrt{2}} X(t-\tau)+\left[\frac{1}{n^{2}} X(t)+\frac{\widetilde{\varepsilon}^{2}}{4} \sin \frac{1}{X(t-\tau)}\right] \frac{\mathrm{d} W(t)}{\mathrm{d} t}
$$

where $\alpha=(5 / 6), t \in[0,2], n \geq 10, f(t, X(t), X(t-\tau))=(1 /$ $n) X(t)+\widetilde{\varepsilon} X(t-\tau), \quad$ and $g(t, X(t), X(t-\tau))=\left(1 / n^{2}\right) X$ $(t)+\left(\widetilde{\varepsilon}^{2} / 4\right) \sin (1 / X(t-\tau))$ are measurable continuous functions, $\widetilde{\varepsilon}$ is an arbitrary number, and $0<\widetilde{\varepsilon}<(\sqrt{2} / 8)$. 
Because

$$
\begin{aligned}
& \|f(t, Z(t), Z(t-\tau))-f(t, X(t), X(t-\tau))\|^{2} \\
& +\|g(t, Z(t), Z(t-\tau))-g(t, X(t), X(t-\tau))\|^{2} \\
& =\left\|\frac{1}{n} Z(t)+\frac{\widetilde{\varepsilon}}{\sqrt{2}} Z(t-\tau)-\frac{1}{n} X(t)-\frac{\widetilde{\varepsilon}}{\sqrt{2}} X(t-\tau)\right\|^{2} \\
& +\left\|\frac{1}{n^{2}} Z(t)+\frac{\widetilde{\mathcal{E}}^{2}}{4} \sin \frac{1}{Z(t-\tau)}-\frac{1}{n^{2}} X(t)-\frac{\widetilde{\mathcal{E}}^{2}}{4} \sin \frac{1}{X(t-\tau)}\right\|^{2} \\
& \leq \frac{4}{n^{2}}\|Z(t)-X(t)\|^{2}+\widetilde{\mathcal{\varepsilon}}^{2}\|Z(t-\tau)-X(t-\tau)\|^{2}+\frac{\widetilde{\mathcal{\varepsilon}}^{4}}{8}\left\|\sin \frac{1}{Z(t-\tau)}-\sin \frac{1}{X(t-\tau)}\right\|^{2} \\
& =\frac{4}{n^{2}}\|Z(t)-X(t)\|^{2}+\widetilde{\mathcal{\varepsilon}}^{2}\|Z(t-\tau)-X(t-\tau)\|^{2} \\
& +\frac{\widetilde{\varepsilon}^{4}}{8}\left\|2 \cos \frac{(1 / Z(t-\tau))+(1 / X(t-\tau))}{2} \sin \frac{(1 / Z(t-\tau))-(1 / X(t-\tau))}{2}\right\|^{2} \\
& \leq \frac{\widetilde{\varepsilon}^{4}}{2}+\frac{4}{n^{2}}\|Z(t)-X(t)\|^{2}+\widetilde{\mathcal{E}}^{2}\|Z(t-\tau)-X(t-\tau)\|^{2} \\
& =G\left(t,\|Z(t)-X(t)\|^{2},\|Z(t-\tau)-X(t-\tau)\|^{2}\right),
\end{aligned}
$$

obviously, $G\left(t,\|Z(t)-X(t)\|^{2},\|Z(t-\tau)-X(t-\tau)\|^{2}\right)$ is nondecreasing, continuous, and concave function and $\widetilde{k}=$ $\max \left\{\sup _{0 \leq t \leq T} b(t), \sup _{0 \leq t \leq T} q(t)\right\}=\max \left(\left(4 / n^{2}\right), \widetilde{\varepsilon}^{2}\right)=(2 / 50)$, $6 \tilde{k} T^{2 \alpha-(1 / 2)} \approx 0.54<(4 \alpha-3)^{(1 / 2)} \Gamma^{2}(\alpha) \approx 0.57$. From the arbitrariness of $\widetilde{\varepsilon}$, we know $G(t, 0,0)=0$. And, $\forall \varepsilon>0, \exists \gamma=$ $(1 / 5 \varepsilon)>0$, such that $\left(3(4 \alpha-3)^{(1 / 2)} T^{2 \alpha}+3(2 \alpha-1) T^{2 \alpha-(1 / 2)} /\right.$ $\left.(2 \alpha-1)(4 \alpha-3){ }^{(1 / 2)} \Gamma^{2}(\alpha)-6 \quad(2 \alpha-1) \tilde{k} T^{2 \alpha-(1 / 2)}\right) . \quad \sup _{0 \leq t \leq T}$ $a(t)<0.20=(1 / 5 \varepsilon) \varepsilon=\gamma \varepsilon$. That satisfies all the conditions of Theorem 2. Therefore, we can conclude that system (82) is Ulam-Hyers stable on $[0,2]$.

Next, we will use a numerical simulation to verify the solution of (82) is Ulam-Hyers stable, and we can see it in Figure 2.

\section{Conclusion}

In this work, the objective is to research the existence and uniqueness of FSDEs with time delays using the novel Caratheodory approximation and the weaker non-Lipschitz condition. Furthermore, different assumptions are used to prove the Ulam-Hyers stability of the solutions. Finally, we present two examples to test the validity of the proposed theory. Our future work will focus on exploring Ulam-Hyers stability of various types of fractional differential equations with weaker conditions, and the explored conditions can be applied to a wider range of differential equations.

\section{Data Availability}

The data used to support the findings of the study are available from the corresponding author upon request.

\section{Conflicts of Interest}

The authors declare that they have no conflicts of interest.

\section{Acknowledgments}

This work was supported by the Natural Science Special Research Fund Project of Guizhou University (202002).

\section{References}

[1] A. Kilbas, H. Srivastava, and J. Trujillo, Theory and Applications of Fractional Differential Equations, North-Holland Mathematics Studies, Vol. 207, Elsevier, Amsterdam, Netherlands, 2006.

[2] Y. Zhou, J. R. Wang, and L. Zhang, Basic Theory of Fractional Differential Equations, World Scientific, Singapore, 2014.

[3] K. S. Miller and B. Ross, An Introduction to the Fractional Calculus and Fractional Differential Equations, a WileyInterscience Publication, Hoboken, NJ, USA, 1993.

[4] I. Podlubny and K. V. Thimann, Fractional Differential Equations: An Introduction to Fractional Derivatives, Fractional Differential Equations, to Methods of Their Solution and Some of Their Applications, Academic Press, Cambridge, UK, 1999. 
[5] R. Agarwal, S. Hristova, and D. O'Regan, Non-instantaneous Impulses in Differential Equations, Springer, Cham, Switzerland, 2017.

[6] A. Pratap, R. Raja, J. Alzabut, J. Cao, G. Rajchakit, and C. Huang, "Mittag-Leffler stability and adaptive impulsive synchronization of fractional order neural networks in quaternion field," Mathematical Methods in the Applied Sciences, vol. 43, no. 10, pp. 6223-6253, 2020.

[7] J. Wang, Y. Zhou, and M. Fečkan, "Abstract Cauchy problem for fractional differential equations," Nonlinear Dynamics, vol. 71, no. 4, pp. 685-700, 2013.

[8] A. Zada, S. Ali, and T. Li, "Analysis of a new class of impulsive implicit sequential fractional differential equations," International Journal of Nonlinear Sciences and Numerical Simulation, vol. 21, no. 6, pp. 571-587, 2020.

[9] G. C. Wu, "A fractional variational iteration method for solving fractional nonlinear differential equations," Computers \& Mathematics with Applications, vol. 61, no. 8, pp. 2186-2190, 2010.

[10] D. F. Luo, A. Zada, S. Shaleena, and M. Ahmad, "Analysis of a coupled system of fractional differential equations with non-separated boundary conditions," Advances in Difference Equations, vol. 2020, Article ID 590, 2020.

[11] A. Pratap, R. Raja, J. D. Cao, J. Alzabut, and C. X. Huang, "Finite-time synchronization criterion of graph theory perspective fractional-order coupled discontinuous neural networks," Advances in Difference Equations, vol. 2020, no. 1, pp. 1-24, 2020.

[12] T. Abdeljawad, R. Amin, K. Shah, Q. Al-Mdallal, and F. Jarad, "Efficient sustainable algorithm for numerical solutions of systems of fractional order differential equations by Haar wavelet collocation method," Alexandria Engineering Journal, vol. 59, no. 4, pp. 2391-2400, 2020.

[13] B. G. Jia, "The asymptotic behavior of Caputo delta fractional equations," Mathematical Methods in the Applied Sciences, vol. 39, no. 18, pp. 5355-5364, 2016.

[14] A. Pratap, R. Raja, G. Rajchakit, J. Cao, and O. Bagdasar, "Mittag-Leffler state estimator design and synchronization analysis for fractional-order BAM neural networks with time delays," International Journal of Adaptive Control and Signal Processing, vol. 33, no. 5, pp. 855-874, 2019.

[15] T. Abdeljawad, R. P. Agarwal, E. Karapinar, and P. S. Kumari, "Solutions of the nonlinear integral equation and fractional differential equation using the technique of a fixed point with a numerical experiment in extended b-metric space," Symmetry, vol. 11, no. 5, 2019.

[16] Q. X. Zhu and T. W. Huang, "Stability analysis for a class of stochastic delay nonlinear systems driven by G-Brownian motion," Systems \& Control Letters, vol. 140, Article ID 104699, 2020.

[17] Q. Zhu, "Stability analysis of stochastic delay differential equations with Lévy noise," Systems \& Control Letters, vol. 118, pp. 62-68, 2018.

[18] R. Raja, U. K. Raja, R. Samidurai, and A. Leelamani, "Passivity analysis for uncertain discrete-time stochastic BAM neural networks with time-varying delays," Neural Computing and Applications, vol. 25, no. 3-4, pp. 751-766, 2014.

[19] Q. Zhu and B. Song, "Exponential stability of impulsive nonlinear stochastic differential equations with mixed delays," Nonlinear Analysis: Real World Applications, vol. 12, no. 5, pp. 2851-2860, 2011.

[20] A. Hojjat, M. Hamidreza, and A. Hassen, "Existence and uniqueness of positive solutions for boundary value problems of fractional differential equations," Filomat, vol. 31, no. 9, pp. 2675-2682, 2017.

[21] D. F. Luo, K. Shah, and Z. G. Luo, "On the novel Ulam-Hyers stability for a class of nonlinear $\psi$ Hilfer fractional differential equation with time-varying delays," Mediterranean Journal of Mathematics, vol. 16, no. 5, Article ID 112, 2019.

[22] J. V. C. Sousa, D. S. Oliveira, and E. Capelas de Oliveira, "On the existence and stability for noninstantaneous impulsive fractional integrodifferential equation," Mathematical Methods in the Applied Sciences, vol. 42, no. 4, pp. 1249-1261, 2019.

[23] F. Chen and Y. Zhou, "Existence and Ulam stability of solutions for discrete fractional boundary value problem," Discrete Dynamics in Nature and Society, vol. 2013, Article ID 459161, 7 pages, 2013.

[24] D. Luo and Z. Luo, "Existence and Hyers-Ulam stability results for a class of fractional order delay differential equations with non-instantaneous impulses," Mathematica Slovaca, vol. 70, no. 5, pp. 1231-1248, 2020.

[25] Y. Guo, X. B. Shu, Y. Li, and F. Xu, "The existence and Hyers-Ulam stability of solution for an impulsive RiemannLiouville fractional neutral functional stochastic differential equation with infinite delay of order $1<\beta<2$," Boundary Value Problems, vol. 2019, Article ID 9372406, 12 pages, 2019.

[26] S. Ulam, A Collection of Mathematical Problem, Interscience, New York, NY, USA, 1960.

[27] D. H. Hyers, "On the stability of the linear functional equation," Proceedings of the National Academy of Sciences, vol. 27, no. 4, pp. 222-224, 1941.

[28] J. Wang and X. Li, "A uniform method to Ulam-Hyers stability for some linear fractional equations," Mediterranean Journal of Mathematics, vol. 13, no. 2, pp. 625-635, 2016.

[29] A. Zada, J. Alzabut, H. Waheed, and P. Ioan-Lucian, "Ulam-Hyers stability of impulsive integrodifferential equations with RiemannLiouville boundary conditions," Advances in Difference Equations, vol. 2020, no. 1, Article ID 64, 2020.

[30] D. F. Luo, Z. G. Luo, and H. J. Qiu, "Existence and Hyers-Ulam stability of solutions for a mixed fractional-order nonlinear delay difference equation with parameters," Mathematical Problems in Engineering, vol. 2020, Article ID 9372406, 12 pages, 2020.

[31] D. Luo, T. Abdeljawad, and Z. Luo, "Ulam-Hyers stability results for a novel nonlinear Nabla Caputo fractional variable-order difference system," Turkish Journal of Mathematics, vol. 45, no. 1, pp. 456-470, 2021.

[32] J. Wang and Y. Zhang, "Ulam-Hyers-Mittag-Leffler stability of fractional-order delay differential equations," Optimization, vol. 63, no. 8, pp. 1181-1190, 2014.

[33] A. Zada, S. Fatima, Z. Ali, J. Xu, and Y. Cui, "Stability results for a coupled system of impulsive fractional differential equations," Mathematics, vol. 7, no. 10, 2019.

[34] T. Sathiyaraj, J. R. Wang, and P. Balasubramaniam, "Ulams stability of Hilfer fractional stochastic differential systems," The European Physical Journal Plus, vol. 134, no. 12, Article ID 605, 2019.

[35] M. Abouagwa and J. Li, "Approximation properties for solutions to It $\hat{o}$ Doob stochastic fractional differential equations with non-Lipschitz coefficients," Stochastics and Dynamics, vol. 19, no. 1, Article ID 1950029, 2019.

[36] A. Ahmadova and N. I. Mahmudov, "Ulam-Hyers stability of Caputo type fractional stochastic neutral differential equations," Statistics and Probability Letters, vol. 168, Article ID 108949, 2021. 
[37] M. Abouagwa, J. Liu, and J. Li, "Carathéodory approximations and stability of solutions to non-Lipschitz stochastic fractional differential equations of Itô-Doob type," Applied Mathematics and Computation, vol. 329, pp. 143-153, 2018.

[38] X. R. Mao, "Approximate solutions for a class of stochastic evolution equations with variable delays," Numerical Functional Analysis and Optimization, vol. 12, no. 5-6, pp. 5-533, 2007.

[39] K. Balasundaram, R. Raja, Q. Zhu, S. Chandrasekaran, and H. Zhou, "New global asymptotic stability of discrete-time recurrent neural networks with multiple time-varying delays in the leakage term and impulsive effects," Neurocomputing, vol. 214, pp. 420-429, 2016.

[40] S. Senthilraj, R. Raja, F. Jiang, Q. Zhu, and R. Samidurai, "New delay-interval-dependent stability analysis of neutral type BAM neural networks with successive time delay components," Neurocomputing, vol. 171, pp. 1265-1280, 2016. 